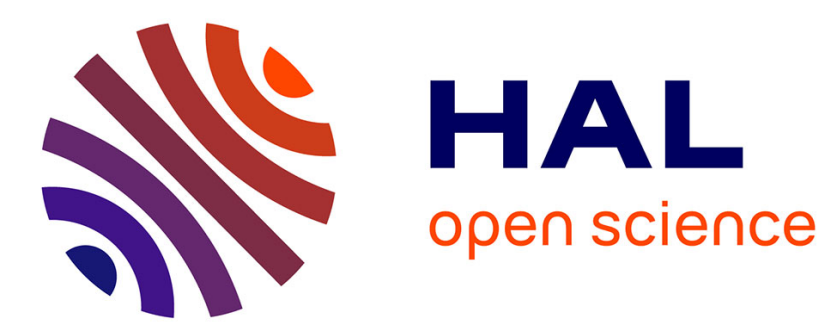

\title{
Automatic Generation of Modular Multipliers for FPGA Applications
}

\author{
Jean-Michel Muller, Jean-Luc Beuchat
}

\section{To cite this version:}

Jean-Michel Muller, Jean-Luc Beuchat. Automatic Generation of Modular Multipliers for FPGA Applications. IEEE Transactions on Computers, 2008, 57 (12), pp.1600-1613. 10.1109/TC.2008.102 . ensl-00122716v3

HAL Id: ensl-00122716

https://hal-ens-lyon.archives-ouvertes.fr/ensl-00122716v3

Submitted on 24 Nov 2008

HAL is a multi-disciplinary open access archive for the deposit and dissemination of scientific research documents, whether they are published or not. The documents may come from teaching and research institutions in France or abroad, or from public or private research centers.
L'archive ouverte pluridisciplinaire HAL, est destinée au dépôt et à la diffusion de documents scientifiques de niveau recherche, publiés ou non, émanant des établissements d'enseignement et de recherche français ou étrangers, des laboratoires publics ou privés. 


\title{
Automatic Generation of Modular Multipliers for FPGA Applications
}

\author{
Jean-Luc Beuchat and Jean-Michel Muller, Senior Member, IEEE
}

\begin{abstract}
Since redundant number systems allow for constant time addition, they are often at the heart of modular multipliers designed for public-key cryptography (PKC) applications. Indeed, PKC involves large operands (160 to 1,024 bits), and several researchers proposed carry-save or borrow-save algorithms. However, these number systems do not take advantage of the dedicated carry logic available in modern Field-Programmable Gate Arrays (FPGAs). To overcome this problem, we suggest to perform modular multiplication in a high-radix carry-save number system, where a sum bit of the carry-save representation is replaced by a sum word. Two digits are then added by means of a small Carry-Ripple Adder (CRA). Furthermore, we propose an algorithm that selects the best high-radix carry-save representation for a given modulus and generates a synthesizable VHDL description of the operator.
\end{abstract}

Index Terms-Modular multiplication, high-radix carry-save number system, FPGA.

\section{INTRODUCTION}

$T^{\prime}$ HIS paper is devoted to the study of modular multiplication of large operands on Field-Programmable Gate Arrays (FPGAs). This operation is crucial in many public-key cryptosystems (e.g., elliptic curve cryptography, XTR, and RSA), and various solutions have already been investigated. Since iterative algorithms offer a good tradeoff between calculation time and circuit area, they have received considerable attention. Least-significant-digit-first schemes are often based on Montgomery's algorithm [1]. However, that approach requires preprocessing and postprocessing and is of interest when a large amount of consecutive modular multiplications is required (e.g., modular exponentiation). In this paper, we will consider a most-significant-digit-first scheme.

\subsection{Horner's Rule-Based Modular Multiplication}

In order to compute $\langle X Y\rangle_{M}=X Y \bmod M$, where $M$ is an $n$-bit integer such that $2^{n-1}<M<2^{n}$, our algorithm is described by an iterative procedure based on the celebrated Horner's rule:

$$
\langle X Y\rangle_{M}=\left\langle\left(\ldots\left(\left(x_{r-1} Y\right) 2+x_{r-2} Y\right) 2+\ldots\right) 2+x_{0} Y\right\rangle_{M},
$$

where $X=x_{r-1} x_{r-2} \ldots x_{1} x_{0}$ is an unsigned $r$-bit integer, and $Y$ is an $n$-bit integer belonging to $\{0, \ldots, M-1\}$. This equation can be expressed recursively as follows (we

- J.-L. Beuchat is with the Graduate School of Systems and Information Engineering, Laboratory of Cryptography and Information Security, University of Tsukuba, 1-1-1 Tennodai, Tsukuba, Ibaraki 305-8573, Japan. E-mail: beuchat@risk.tsukuba.ac.jp.

- J.-M. Muller is with CNRS, projet INRIA Arénaire, Université de Lyon, Laboratoire LIP, ENS Lyon, 46 allée d'Italie, 69364 Lyon Cédex 07, France. E-mail: Jean-Michel.Muller@ens-lyon.fr.

Manuscript received 1 Jan. 2007; revised 28 Apr. 2008; accepted 19 May 2008; published online 27 June 2008.

Recommended for acceptance by M. Schulte.

For information on obtaining reprints of this article, please send e-mail to: tc@computer.org, and reference IEEECS Log Number TC-0003-0107.

Digital Object Identifier no. 10.1109/TC.2008.102. perform a modulo $M$ reduction at each step in order to keep an $n$-bit intermediate result):

$$
\begin{aligned}
T[i] & =2 Q[i+1]+x_{i} Y, \\
Q[i] & =\langle T[i]\rangle_{M},
\end{aligned}
$$

where $Q[r]=0$, and $Q[0]=\langle X Y\rangle_{M}$. Since $Q[i+1]$ and $Y$ are less than or equal to $M-1, T[i]<3 M$, and (1) is implemented by means of a left shift, an addition, a comparator, and up to two subtractions to perform the modulo $M$ reduction [2].

Since public-key cryptography involves large integers, operands are often represented in the carry-save number system, which enables addition in constant time (see, for instance, [3]). However, due to the redundancy of this representation, comparison requires a conversion in a nonredundant number system. This operation involves carry propagations, thus losing the main advantage of the carrysave representation. Several improvements of the algorithm sketched by (1) have therefore been investigated to avoid comparisons. They are based on the following observation: computing a number $P[i]$ congruent to $Q[i]$ modulo $M$ only requires inspecting a few most significant digits of $T[i]$. In order to avoid an expensive final modular reduction, $P[i]$ should be less than a very small multiple of $M$. The iteration described in this paper returns, for instance, $\langle X Y\rangle_{M}$ or $\langle X Y\rangle_{M}+M$ and requires at most one subtraction to get the final result.

Koç and Hung [4], [5] proposed, for instance, a carrysave algorithm based on a sign estimation technique. At each step, $-M, 0$, or $M$ is added to $T[i]$ according to a few most significant digits of $Q[i+1]$. Takagi and Yajima [6], [7] applied a similar technique to design signed-digit-based architectures. When the modulus $M$ is known at design time, which is often the case in publickey cryptography, another approach consists of building 


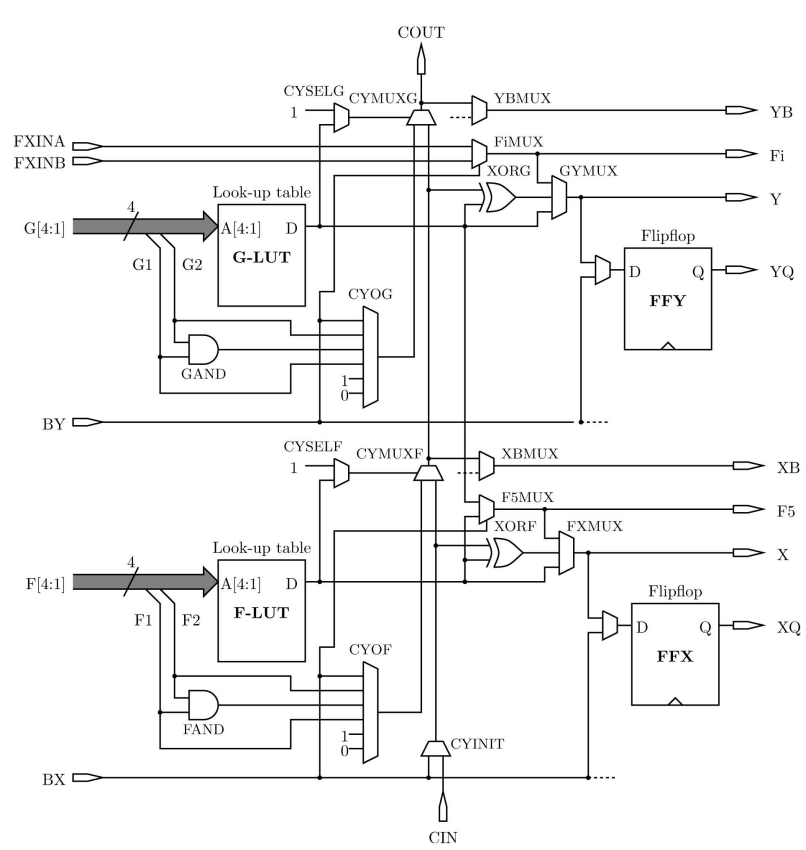

Fig. 1. Simplified diagram of a slice of a Spartan-3 FPGA.

a table $\psi(a)=\left\langle a \cdot 2^{\beta}\right\rangle_{M}$ and in defining the following iteration:

$$
\begin{gathered}
T[i]=2 P[i+1]+x_{i} Y, \\
P[i]=\psi\left(T[i] \operatorname{div} 2^{\beta}\right)+\langle T[i]\rangle_{2^{\beta}},
\end{gathered}
$$

where $P[r]=0$, and $\beta$ is generally chosen to be equal to $n$ or $n-1$. Since $\psi\left(T[i] \operatorname{div} 2^{\beta}\right)$ is an $n$-bit number, $P[i]$ and $T[i]$ are respectively $(n+1)$ - and $(n+3)$-bit numbers. Therefore, the algorithm sketched by the above equations requires a small table. Carry-save implementations of (2) and (3) have, for example, been proposed by Jeong and Burleson [8], Kim and Sobelman [9], and Peeters et al. [10]. Since these algorithms depend on the modulus $M$, they seem likely candidates for cryptographic hardware based on FPGAs: the reconfigurability of these devices allows one to optimize the architecture according to some parameters (e.g., the modulus) and to modify the hardware whenever they change.

\subsection{FPGA-Specific Issues}

Modern FPGAs are mainly designed for digital signal processing applications involving rather small operands (16 to 32 bits). FPGA manufacturers chose to include dedicated carry logic enabling the implementation of fast carry-ripple adders (CRAs) for such operand sizes. Let us study, for example, the architecture of a Spartan-3 device. Fig. 1 describes the simplified architecture of a slice, which is the main logic resource for implementing synchronous and combinatorial circuits. Each slice embeds two fourinput function generators (G-LUT and F-LUT), two storage elements (i.e., flip-flops FFY and FFX), carry logic (CYSELG, CYMUXG, CYSELF, CYMUXF, and CYINIT), arithmetic gates (GAND, FAND, XORG, and XORF), and widefunction multiplexers. Each function generator is implemented by means of a programmable lookup table (LUT). Recall that a full-adder (FA) cell has two bits $x_{i}$ and $y_{i}$, as well as a carry-in bit $c_{\mathrm{in}}$, as inputs and computes a sum bit $s_{i}$
TABLE 1

Area and Number of LUTs of Three Carry-Save Iteration Stages

\begin{tabular}{|c|c|c|c|c|}
\hline \multirow{2}{*}{ Algorithm } & \multicolumn{2}{|c|}{ Without carry logic } & \multicolumn{2}{|c|}{ With carry logic } \\
\hline & $n=32$ & $n=64$ & $n=32$ & $n=64$ \\
\hline Jeong & 107 slices & 210 slices & 119 slices & 232 slices \\
\hline Burleson [8] & 200 LUTs & 392 LUTs & 141 LUTs & 271 LUTs \\
\hline Kim and & 74 slices & 166 slices & 93 slices & 188 slices \\
\hline Sobelman [9] & 139 LUTs & 268 LUTs & 123 LUTS & 249 LUTs \\
\hline Peeters & 74 slices & 160 slices & 95 slices & 190 slices \\
\hline et al. [10] & 140 LUTs & 271 LUTs & 95 LUTs & 190 LUTs \\
\hline
\end{tabular}
(Spartan-3 FPGA)

and a carry-out bit $c_{\text {out }}$ such that $2 c_{\text {out }}+s_{i}=x_{i}+y_{i}+c_{\text {in }}$. Let $h_{i}=x_{i} \oplus y_{i}$. Then, we have

$$
\begin{gathered}
s_{i}=h_{i} \oplus c_{\text {in }}, \\
c_{\text {out }}= \begin{cases}x_{i}, & \text { if } \left.h_{i}=0 \text { (i.e., } x_{i}=y_{i}\right), \\
c_{\text {in }}, & \text { otherwise. }\end{cases}
\end{gathered}
$$

Assume that the F-LUT function generator computes $h_{i}$. Then, the XORF gate implements (4), whereas (5) involves three multiplexers (CYOF, CYSELF, and CYMUXF). $s_{i}$ is either sent to another slice (output X) or stored in a flip-flop (FFX). The G-LUT function generator allows one to implement a second FA cell within the same slice, which thus embeds a 2-bit CRA. Unfortunately, a conventional carrysave adder (CSA) requires twice as many hardware resources: since each slice has a single-input carry CIN, it is impossible to implement two FA cells with independent carryin bits. Therefore, hardware design tools allocate two function generators when they are provided with a VHDL description of (4) and (5). It is of course possible to write a VHDL code that explicitly instantiates F-LUT, XORF, and CYMUXF. In this case, note that G-LUT can only be used to implement the control unit or the $\psi($.) table of (3). According to experiment results, G-LUT often remains unused. Though reducing the number of LUTs of the design, taking advantage of dedicated logic to describe a CSA leads to a larger operator (Table 1). Similar problems arise, for instance, on all Virtex devices (Xilinx) and Cyclone II FPGAs (Altera). It is therefore interesting to investigate modular multiplication algorithms based on FPGA-friendly number systems.

\subsection{Our Contribution}

We proposed a family of radix-2 algorithms designed for FPGAs embedding four-input LUTs and dedicated carry logic in [11]. Table 2 compares our iteration stage against

TABLE 2

Area and Delay of Carry-Save and Radix-2 Iteration Stages (Spartan-3 FPGA)

\begin{tabular}{|l||l|l|l|}
\hline \multicolumn{1}{|c||}{ Algorithm } & $\mathbf{n = 1 6}$ & \multicolumn{1}{c|}{$\boldsymbol{n = 3 2}$} & \multicolumn{1}{c|}{$\boldsymbol{n = 6 4}$} \\
\hline \hline \multirow{2}{*}{ Jeong and Burleson [8] } & 58 slices & 127 slices & 236 slices \\
& $9 \mathrm{~ns}$ & $11 \mathrm{~ns}$ & $14 \mathrm{~ns}$ \\
\hline \multirow{2}{*}{ Kim and Sobelman [9] } & 41 slices & 79 slices & 150 slices \\
& $8 \mathrm{~ns}$ & $10 \mathrm{~ns}$ & $12 \mathrm{~ns}$ \\
\hline \multirow{2}{*}{ Peeters et al. [10] } & 50 slices & 86 slices & 163 slices \\
& $8 \mathrm{~ns}$ & $11 \mathrm{~ns}$ & $12 \mathrm{~ns}$ \\
\hline \multirow{2}{*}{ Beuchat and Muller [11] } & 21 slices & $40 \mathrm{slices}$ & 80 slices \\
& $12 \mathrm{~ns}$ & $14 \mathrm{~ns}$ & $20 \mathrm{~ns}$ \\
\hline
\end{tabular}




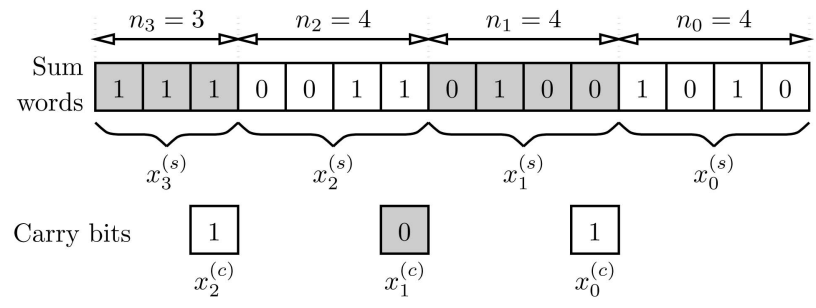

(a)



(b)

Fig. 2. High-radix carry-save numbers. (a) Encoding of the number $X=33,626$. (b) Encoding of $Z=2 X$.

three carry-save schemes. Since these results do not include the conversion from carry-save to unsigned integer that occurs at the end of each multiplication, both the area and delay of carry-save operators are underestimated. According to this experiment, our previous approach is efficient for moduli up to 32 bits. Thus, the aim of this paper is to extend our work to larger moduli. In order to benefit from dedicated carry logic available in almost all FPGA families, we suggest to choose a high-radix carry-save number system, where each sum bit of the carry-save representation is replaced by a sum word (Section 2). Such a number system allows for the design of a modular multiplication algorithm based on small CRAs (Section 3). The main originality of our approach is to analyze the modulus $M$ in order to select the most efficient high-radix carry-save representation and to automatically generate the VHDL description of the operator (Section 4). Experimental results validate the efficiency of the proposed modular multiplication scheme (Section 5). We proposed a preliminary version of this work based on a different iteration in [12].

\section{High-Radix Carry-Save Numbers}

A $k$-digit high-radix carry-save number $X$ is denoted by

$$
X=\left(x_{k-1}, \ldots, x_{0}\right)=\left(\left(x_{k-1}^{(c)}, x_{k-1}^{(s)}\right), \ldots,\left(x_{0}^{(c)}, x_{0}^{(s)}\right)\right),
$$

where the $j$ th digit $x_{j}$ consists of an $n_{j}$-bit sum word $x_{j}^{(s)}$ and a carry bit $x_{j}^{(c)}$ such that $x_{j}=x_{j}^{(s)}+x_{j}^{(c)} 2^{n_{j}}$. According to this definition, we have

$$
\begin{aligned}
X & =x_{0}+x_{1} 2^{n_{0}}+x_{2} 2^{n_{0}+n_{1}}+\cdots+x_{k-1} 2^{n_{0}+\cdots+n_{k-2}} \\
& =x_{0}^{(s)}+\sum_{i=0}^{k-2}\left(x_{i}^{(c)}+x_{i+1}^{(s)}\right) 2^{\sum_{j=0}^{i} n_{i}}+x_{k-1}^{(c)} 2^{\sum_{j=0}^{k-1} n_{i}} .
\end{aligned}
$$

Let us define

$$
X^{(s)}=x_{0}^{(s)}+x_{1}^{(s)} 2^{n_{0}}+\cdots+x_{k-1}^{(s)} 2^{n_{0}+\cdots+n_{k-2}},
$$

and

$$
X^{(c)}=x_{0}^{(c)} 2^{n_{0}}+x_{1}^{(c)} 2^{n_{0}+n_{1}}+\cdots+x_{k-1}^{(c)} 2^{n_{0}+\cdots+n_{k-1}} .
$$

With this notation, we have $X=X^{(s)}+X^{(c)}$. Such a number system has nice properties to deal with large numbers on FPGA:

- Its redundancy allows one to perform addition in constant time (the critical path only depends on $\max _{0 \leq j \leq k-1} n_{j}$ ).
- The addition of a sum word $x_{j}^{(s)}$, a carry bit $x_{j-1}^{(c)}$, and an $n_{j}$-bit unsigned binary number can be performed by a CRA.

A key observation is that all sum words do not need to have the same width. This peculiarity will allow us to select a number system according to the modulus to optimize our operators (Section 4). In the following, we will assume that the carry bit of the most significant digit is always equal to zero (the weight of the most significant carry bit is therefore equal to $2^{n_{0}+n_{1}+\cdots+n_{k-2}}$ ).

Example 1. Fig. 2a describes a four-digit high-radix carrysave number with $n_{0}=n_{1}=n_{2}=4$ and $n_{3}=3$. According to the first definition of this number system, we have

$$
\begin{aligned}
X= & x_{0}^{(s)}+\left(x_{0}^{(c)}+x_{1}^{(s)}\right) \cdot 2^{4}+\left(x_{1}^{(c)}+x_{2}^{(s)}\right) \cdot 2^{8} \\
& +\left(x_{2}^{(c)}+x_{3}^{(s)}\right) \cdot 2^{12} \\
= & 10+(1+4) \cdot 2^{4}+(0+3) \cdot 2^{8}+(1+7) \cdot 2^{12} \\
= & 33,626 .
\end{aligned}
$$

We can also compute

$$
X^{(s)}=10+4 \cdot 2^{4}+3 \cdot 2^{8}+7 \cdot 2^{12}=29,514,
$$

and

$$
X^{(c)}=1 \cdot 2^{4}+0 \cdot 2^{8}+1 \cdot 2^{12}=4,112 .
$$

We obtain $X=X^{(s)}+X^{(c)}=33,626$.

Consider the modular multiplication described by (2) and (3) and assume that both $T[i]$ and $P[i]$ are high-radix carry-save numbers. Each equation involves now the addition of a high-radix carry-save number and an unsigned integer (a partial product $x_{i} Y$ or a number $\psi\left(T[i] \operatorname{div} 2^{\beta}\right.$ ) stored in the table). Fig. 3 describes how to perform these operations: the integer operand is split into $k$ words of

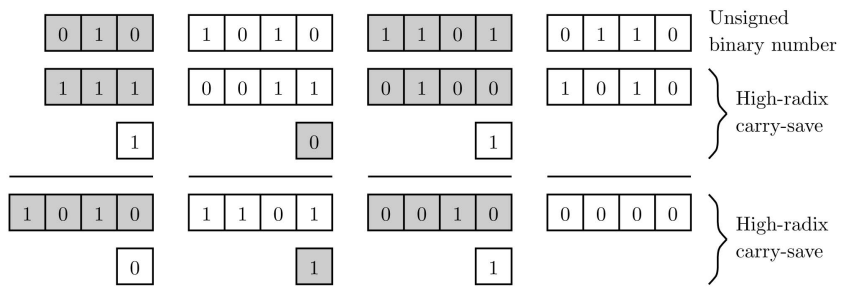

Fig. 3. Addition of an unsigned binary number and a high-radix carrysave number. 
respective lengths $n_{0}, \ldots, n_{k-1}$; then, each of these words is added to a sum word and a carry bit by means of an $n_{j}$-bit CRA. The high-radix carry-save encoding has unfortunately a drawback in the sense that shifting an operand modifies its representation. The following example illustrates this problem, which occurs in the computation of $T[i]$ (2).

Example 2. Let us consider again the number $X=33,626$, whose format is defined in Fig. 2a. By shifting $X$, we obtain $Z=2 X=67,252$ (Fig. 2b). However, the least significant sum word is now a 5-bit number, and

$$
\begin{aligned}
Z & =z_{0}+z_{1} 2^{n_{0}+1}+z_{2} 2^{n_{0}+n_{1}+1}+z_{3} 2^{n_{0}+n_{1}+n_{2}+1} \\
& =20+(1+4) \cdot 2^{5}+(0+3) \cdot 2^{9}+(1+7) \cdot 2^{13} \\
& =67,252 .
\end{aligned}
$$

According to this example, $P[i+1]$ and $P[i]$ do not have the same encoding, and the width of the CRA dealing with the least significant digit of $P$ would increase at each step. The solution consists of converting $T[i]$ to the format of $P[i]$. In the following, we describe a modular multiplication algorithm that minimizes the hardware overhead induced by this conversion.

\section{High-Radix CarRy-Save Modular MULTIPLICATION}

This section describes how to take advantage of a highradix carry-save number system to perform a modular multiplication. We assume the following:

- The modulus $M$ is an $n$-bit number belonging to $\left\{2^{n-1}+1, \ldots, 2^{n}-1\right\}$.

- $X$ is an $r$-bit unsigned integer.

- $\quad Y$ is an unsigned integer smaller than $M$.

- $\alpha$ is a small integer parameter that will determine the size of the table required for the modulo $M$ reduction.

- The most significant sum word of $P[i]$ contains at least $n_{k-1}=5$ bits if $\alpha=1$ and $n_{k-1}=6$ bits if $\alpha=2$. These hypotheses guarantee that

- $P^{(c)}[i]$ is smaller than $2^{n-\alpha}$, i.e. $\left\langle P^{(c)}[i]\right\rangle_{2^{n-\alpha}}=$ $P^{(c)}[i]$ (we fixed the carry bit of the most significant digit of a high-radix carry-save number to zero in Section 2), and

- $\quad P^{(s)}[i]$ is an $(n+2)$-bit number (a proof is given in Appendix A).

- At each iteration, we compute a high-radix carrysave number $P[i]$ congruent to $2 P[i+1]+x_{i} Y$ modulo $M$.

According to our hypotheses, we have

$$
\begin{aligned}
P[i+1]= & \left(P^{(s)}[i+1] \operatorname{div} 2^{n-\alpha}\right) \cdot 2^{n-\alpha} \\
& +\left\langle P^{(s)}[i+1]\right\rangle_{2^{n-\alpha}}+\left\langle P^{(c)}[i+1]\right\rangle_{2^{n-\alpha}} \\
= & \left(P^{(s)}[i+1] \operatorname{div} 2^{n-\alpha}\right) \cdot 2^{n-\alpha} \\
& +\left\langle P^{(s)}[i+1]\right\rangle_{2^{n-\alpha}}+P^{(c)}[i+1] .
\end{aligned}
$$

The iteration of our algorithm is slightly different from the one described in Section 1 . Let us define $\rho[i+1]=$ $P^{(s)}[i+1] \operatorname{div} 2^{n-\alpha}$ and write the intermediate result at step $i+1$ as follows:

$$
P[i+1]=\rho[i+1] 2^{n-\alpha}+\left\langle P^{(s)}[i+1]\right\rangle_{2^{n-\alpha}}+P^{(c)}[i+1] .
$$

It is worth noticing that according to our hypotheses, $\rho[i+1]$ is a 3 - or 4-bit unsigned number for $\alpha=1$ or $\alpha=2$, respectively. Thus, a small table addressed by $\rho[i+1]$ allows one to efficiently compute a number congruent to $P[i+1]$ modulo $M$ :

$$
\begin{aligned}
P[i+1] \equiv & \left\langle\rho[i+1] 2^{n-\alpha}\right\rangle_{M}+\left\langle P^{(s)}[i+1]\right\rangle_{2^{n-\alpha}} \\
& +P^{(c)}[i+1](\bmod M) .
\end{aligned}
$$

Note that when $\alpha=1$, the table can be stored within the LUTs of a CRA on Spartan-3 and Virtex FPGAs [11]. Since we compute a high-radix carry-save number congruent to $X Y$ modulo $M$, a conversion and a final modulo $M$ reduction are mandatory. In order to keep the hardware overhead as small as possible, we apply a trick proposed by Peeters et al. [10] in the case of a carry-save implementation. At each step, our algorithm computes

$$
\begin{aligned}
P[i]= & x_{i} Y+2\left\langle\rho[i+1] 2^{n-\alpha}\right\rangle_{M} \\
& +2\left(\left\langle P^{(s)}[i+1]\right\rangle_{2^{n-\alpha}}+P^{(c)}[i+1]\right) .
\end{aligned}
$$

According to this equation, $P[i]$ is always even when $x_{i} Y=0$. Thus, by performing an additional step with $x_{-1}=0$, we obtain an even number $P[-1]$ congruent to $2 X Y$ modulo $M$. Note that $P[-1] / 2$ is smaller than or equal to $P[0]$ and easy to compute (a right shift of one position involves only wiring). Furthermore, performing the final modulo $M$ correction with $P[-1] / 2$ requires fewer hardware resources. Let us define

$$
\psi_{\text {max }}= \begin{cases}\max _{0 \leq j<2^{4}}\left\langle j \cdot 2^{n-2}\right\rangle_{M}, & \text { if } \alpha=2 \text { and } n_{k-1} \geq 5, \\ \max _{0 \leq j<2^{3}}\left\langle j \cdot 2^{n-1}\right\rangle_{M}, & \text { if } \alpha=1 \text { and } n_{k-1} \geq 6 .\end{cases}
$$

$P[-1] / 2$ is a high-radix carry-save number equal to $\langle X Y\rangle_{M}$ or $\langle X Y\rangle_{M}+M$, and the final modulo $M$ reduction requires at most one subtraction in the following cases: ${ }^{1}$

- $\quad \alpha=2$ and $n_{k-1} \geq 5$.

- $\alpha=1, n_{k-1} \geq 6$, and

$$
\frac{2^{n-1}-1+2^{n_{0}}+\cdots+2^{n_{0}+\cdots+n_{k-2}}+\psi_{\max }}{2}<M .
$$

A proof of correctness of this modular multiplication scheme, summarized by Algorithm 1, is provided in Appendix A. At each iteration, a new intermediate result $P[i]$ is computed in two steps. Let $\tilde{P}[i+1]$ be a high-radix carry-save number such that $\tilde{P}^{(s)}[i+1]=\left\langle P^{(s)}[i+1]\right\rangle_{2^{n-\alpha}}$ and $\tilde{P}^{(c)}[i+1]=P^{(c)}[i+1]$. We first carry out the sum

1. Note that the algorithm described in our preliminary work [12] does not satisfy this property, and the final modular reduction depends on the high-radix number system and the modulus $M$. 
of the partial product $x_{i} Y$ and $2 \tilde{P}[i+1]$ by means of small CRAs:

$$
T[i]=2 \tilde{P}[i+1]+x_{i} Y .
$$

By shifting the high-radix carry-save number $P[i+1]$, we define a new internal representation for $T[i]$ (Section 2). The second step consists of adding $2\left\langle\rho[i+1] \cdot 2^{n-\alpha}\right\rangle_{M}$ to $T[i]$ and converting the result to the format of $P[i+1]$.

\section{Algorithm 1. High-radix carry-save modulo $M$ multiplication}

Input: An $n$-bit modulus $M$ such that $2^{n-1}<M<2^{n}$, an $r$-bit number $X \in \mathbb{N}, Y \in\{0, \ldots, M-1\}$, and a parameter $\alpha \in\{1,2\} . P[i]$ and $T[i]$ are high-radix carry-save numbers.

Output: $P=\langle X Y\rangle_{M}$.

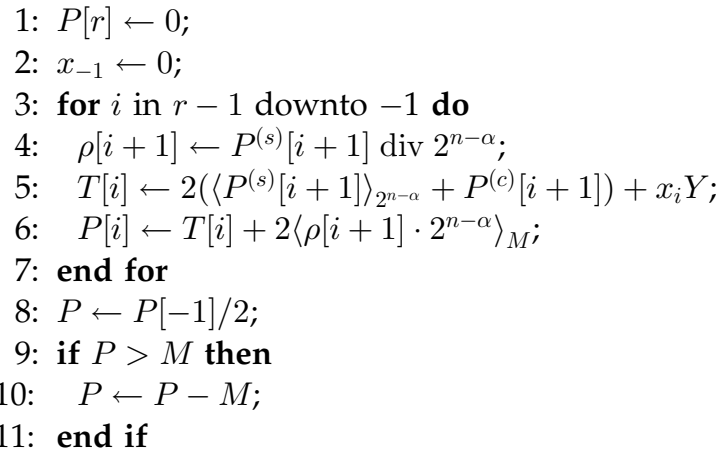

The main difficulty of the implementation arises from the left shift of the carry bits $P^{(c)}[i+1]$. Since $T[i]$ has a different encoding, it is necessary to perform a conversion. We suggest to compute a high-radix carry-save number $U[i]$ that has the same encoding as $P[i+1]$, and is equal to the sum of the carry bits of $T[i]$ and the output of the table (i.e. $\left.2\left\langle\rho[i+1] \cdot 2^{n-\alpha}\right\rangle_{M}\right)$. Therefore, we perform the following operations at each iteration of Algorithm 1:

$$
\begin{aligned}
& T[i] \leftarrow 2 \tilde{P}[i+1]+x_{i} Y, \\
& U[i] \leftarrow 2\left\langle\rho[i+1] \cdot 2^{n-\alpha}\right\rangle_{M}+T^{(c)}[i], \\
& P[i] \leftarrow U[i]+T^{(s)}[i] .
\end{aligned}
$$

Example 3. Let $n=16, k=4$, and $n_{0}=n_{1}=n_{2}=n_{3}=4$. The high-radix carry-save number $T[i]$ contains three carry bits of respective weights $2^{5}, 2^{9}$, and $2^{13}$ (recall the constraint introduced in Section 2: the carry bit of the most significant digit is always equal to zero). We split $2\left\langle\rho[i+1] \cdot 2^{n-\alpha}\right\rangle_{M}$ into four 4-bit words and perform three additions to compute $U[i]$ (Fig. 4).

\section{Choice of a High-Radix Carry-Save Number SYSTEM}

Let us represent the table involved in the modulo $M$ correction by a matrix $\Psi$. Each line $\psi_{\rho}$ of $\Psi$ stores an $n$-bit number $\left\langle\rho[i+1] \cdot 2^{n-\alpha}\right\rangle_{M}$. In the following, we will have to consider a subset of consecutive columns of $\Psi$. Let $\Psi^{(j+h: j)}$ be the matrix constituted by columns $j$ to $j+h$ of $\Psi$. Each line of $\Psi^{(j+h: j)}$ contains an $(h+1)$-bit number $\psi_{\rho}^{(j+h: j)}$.

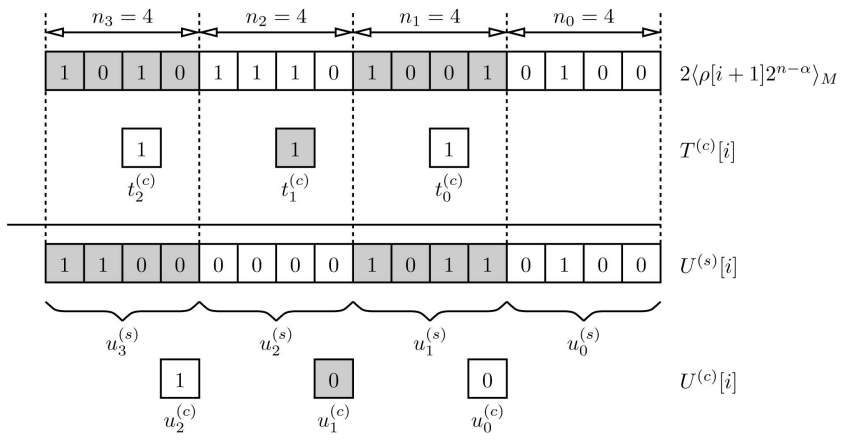

Fig. 4. Conversion of $T[i]$ by merging its carry bits with $2\left\langle\rho[i+1] \cdot 2^{n-\alpha}\right\rangle_{M}$.

Example 4. Let us consider the 16-bit modulus $M=54,107$ and assume that $\alpha=1$. We have

$$
\Psi=\left[\begin{array}{llllllllllllllll}
0 & 0 & 0 & 0 & 0 & 0 & 0 & 0 & 0 & 0 & 0 & 0 & 0 & 0 & 0 & 0 \\
1 & 0 & 0 & 0 & 0 & 0 & 0 & 0 & 0 & 0 & 0 & 0 & 0 & 0 & 0 & 0 \\
0 & 0 & 1 & 0 & 1 & 1 & 0 & 0 & 1 & 0 & 1 & 0 & 0 & 1 & 0 & 1 \\
1 & 0 & 1 & 0 & 1 & 1 & 0 & 0 & 1 & 0 & 1 & 0 & 0 & 1 & 0 & 1 \\
0 & 1 & 0 & 1 & 1 & 0 & 0 & 1 & 0 & 1 & 0 & 0 & 1 & 0 & 1 & 0 \\
0 & 0 & 0 & 0 & 0 & 1 & 0 & 1 & 1 & 1 & 1 & 0 & 1 & 1 & 1 & 1 \\
1 & 0 & 0 & 0 & 0 & 1 & 0 & 1 & 1 & 1 & 1 & 0 & 1 & 1 & 1 & 1 \\
0 & 0 & 1 & 1 & 0 & 0 & 1 & 0 & 1 & 0 & 0 & 1 & 0 & 1 & 0 & 0
\end{array}\right] .
$$

According to our notation, we have, for instance

$$
\Psi^{(6: 3)}=\left(\psi_{\rho}^{(6: 3)}\right)=\left[\begin{array}{cccc}
0 & 0 & 0 & 0 \\
0 & 0 & 0 & 0 \\
1 & 0 & 0 & 1 \\
1 & 0 & 0 & 1 \\
0 & 0 & 1 & 0 \\
1 & 0 & 1 & 1 \\
1 & 0 & 1 & 1 \\
0 & 1 & 0 & 1
\end{array}\right]
$$

It is worth noticing that the amount of hardware required to compute $U[i]$ depends on the modulus $M$ and the encoding of $P[i]$. For instance, if a column of $\Psi$ contains only zeros, it can be replaced by a carry bit at no extra cost. We propose an algorithm that selects a high-radix carrysave number system minimizing the hardware overhead introduced by the computation of $U[i]$ (6). Assume that we want to merge $t_{w}^{(c)}$ with the $j$ th column and $t_{w+1}^{(c)}$ with the $(j+h)$ th column of $\Psi$, and recall that the carry bits of $T[i]$ are left-shifted compared to those of $P[i]$ and $U[i]$ (Fig. 5). Therefore, we compute a digit $u_{w+1}$ such that

$$
u_{w+1}^{(c)} 2^{h}+u_{w+1}^{(s)}=2(\underbrace{\psi_{\rho}^{(j+h-2: j)}}_{h-1 \text { bits }}+t_{w}^{(c)})+\psi_{\rho}^{(j-1: j-1)} .
$$

This operation involves at most an $(h-1)$-bit CRA. However, it is unlikely that there are very long strings of consecutive ones in matrix $\psi$ (see below). Let us denote by $\#\left(\psi_{\rho}^{(j+h-2: j)}\right)$ the length of the longest string of consecutive ones starting from the least significant bit of $\psi_{\rho}^{(j+h-2: j)}$. Then, the following cases may occur according to $\ell=\max _{\rho} \#\left(\psi_{\rho}^{(j+h-2: j)}\right)$ : 


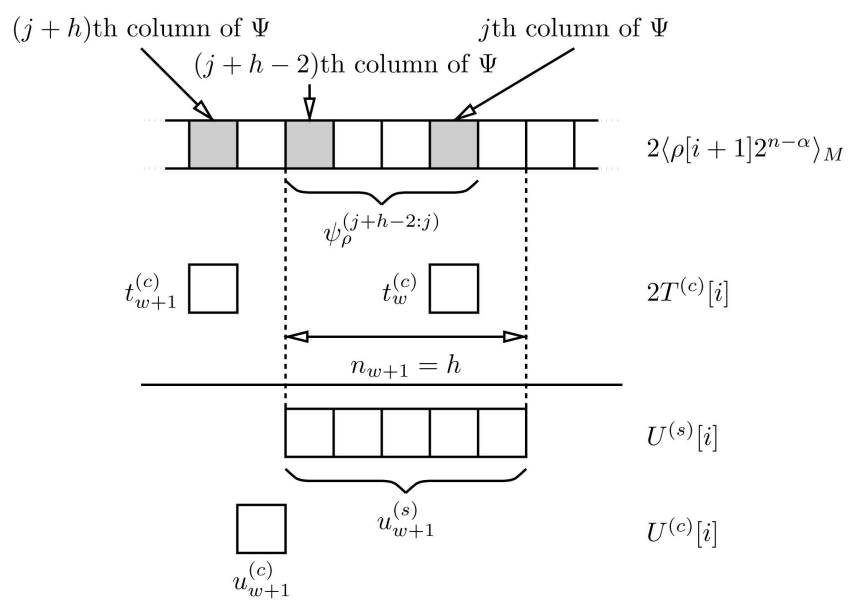

Fig. 5. Cost of the addition of a carry bit (1). In this example, $h$ is equal to five.

- If $\ell=0$, the $j$ th column of $\Psi$ contains only zeros and can be replaced by $t_{w}^{(c)}$ at no extra cost. More formally, we have (Fig. 6a):

$$
\begin{aligned}
& u_{w+1}^{(s)}=4 \psi_{\rho}^{(j+h-2: j+1)}+2 t_{w}^{(c)}+\psi_{\rho}^{(j-1: j-1)}, \\
& u_{w+1}^{(c)}=0 .
\end{aligned}
$$

- If $\ell=h-1$, the addition requires an $(h-1)$-bit CRA, which generates an output carry bit $u_{w+1}^{(c)}$ (see (8) and Fig. 6b). Since this bit will be added to a few bits of $T^{(s)}[i]$ in order to compute $p_{w+2}^{(s)}[i]$, we raise a flag that indicates this carry propagation.

- When $0<\ell<h-1$, an $\ell$-bit CRA computes the sum and generates an output carry $c_{\text {out }}$. If the $(j+\ell)$ th column of $\Psi$ stores only zeros, we replace it by $c_{\text {out }}$ (Fig. 6c). Otherwise, we need an OR gate to add $c_{\text {out }}$ to the $(j+\ell)$ th column. Since we target FPGA applications, a more efficient solution consists of taking advantage of the dedicated carry logic to perform this operation, and we add $t_{w}^{(c)}$ to $\psi_{\rho}^{(j+\ell: j)}$ by means of an ( $\ell+1$ )-bit CRA (Fig. 6d). Note that $u_{w+1}^{(c)}$ is always equal to zero.

Let us try to estimate what values of $\ell$ we can expect in practice. If we consider that the bits in matrix $\psi$ can be viewed as "random" bits, with equal probability for zero as for one, then the average value of $\ell$ will be $N(h) / 2^{h-1}$, where $N(h)$ is the sum of the lengths of the strings of ones that start from the rightmost bit in all possible $(h-1)$-bit strings. Since we obviously have $N(1)=1$ and $N(h)=2 N(h-1)+1$, we immediately deduce that the average value of $\ell$ is $1-1 / 2^{h-1}$ (i.e., less than one).

Example 5 (Example 4 continued). Assume that we want to add carry bits to the third and eighth columns of $\Psi$ (i.e., $j=3$ and $h=5)$. We have to consider the matrix $\Psi^{(6: 3)}$ given by (7) and easily determine that $\ell=2$. Thus, we have to examine the third column of $\Psi^{(6: 3)}$ in order to compute the width of the CRA. Since this column contains a non-null element, we need an $(\ell+1)$-bit CRA (see Fig. 6d).

Let us now build a directed acyclic graph as follows:

- Each node represents a column of the matrix $\Psi$.

- The weight of the edge between nodes $j$ and $j+h$ is given by the width of the CRA responsible for the addition of $\psi_{\rho}^{(j+h-2: j)}$ and $t_{w}^{(c)}(8)$, as well as the flag that indicates a carry propagation.

A shortest path of this graph defines the high-radix carrysave representation minimizing the hardware overhead introduced by the computation of $U[i]$ for a given modulus $M$. The algorithm requires two parameters to control the size of the CRAs:

- Since we want to perform a modular multiplication by means of small CRAs, we have to provide the algorithm with a constraint on the maximal number of positions $w_{\max }$ between two consecutive carry bits (without this constraint, we would, for instance, have an edge from the first node to the last node).

- The minimal distance between two consecutive carries $w_{\min }$ should be greater than or equal to two. It guarantees that the smallest building block is a 2-bit CRA.

Algorithm 2 describes a way to build this graph. After having computed $\Psi$, we have to determine to which columns the most significant bit of $T[i]$ can be added. We denote by $j_{\max }$ the upper index. Recall that when $\alpha=2$, we assume that the most significant sum word of $P[i]$ contains at least $n_{k-1}=5$ bits. Thus, we deduce that $j_{\max }=n-2$

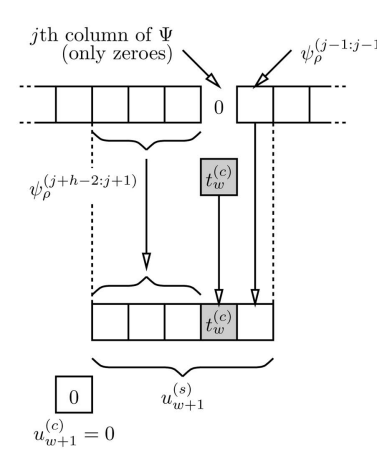

(a)

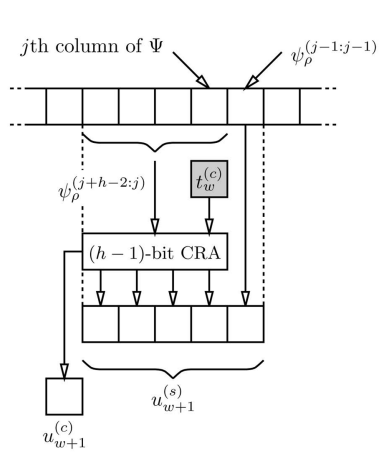

(b)

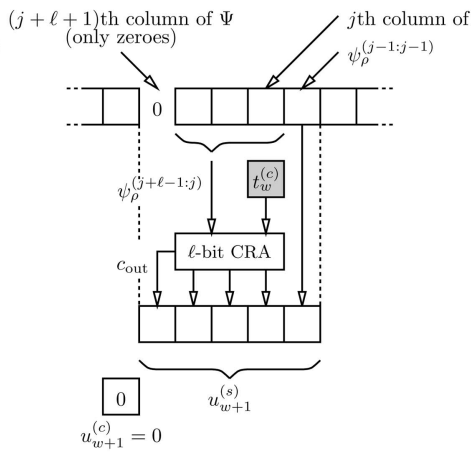

(c)

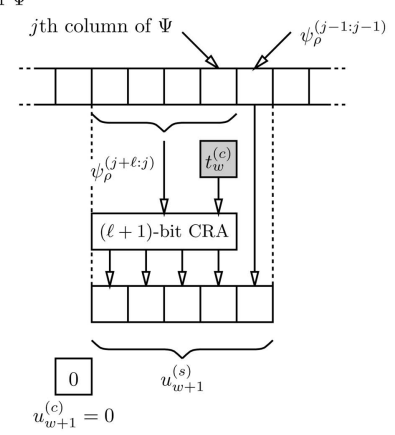

(d)

Fig. 6. Cost of the addition of a carry bit (2). (a) Only zeros in the $j$ th column of $\Psi$. (b) $\ell=h-1$. (c) $\ell<h-1$ and the $(j+\ell+1)$ th column of $\Psi$ contains only zeros. (d) $\ell<h-1$. 


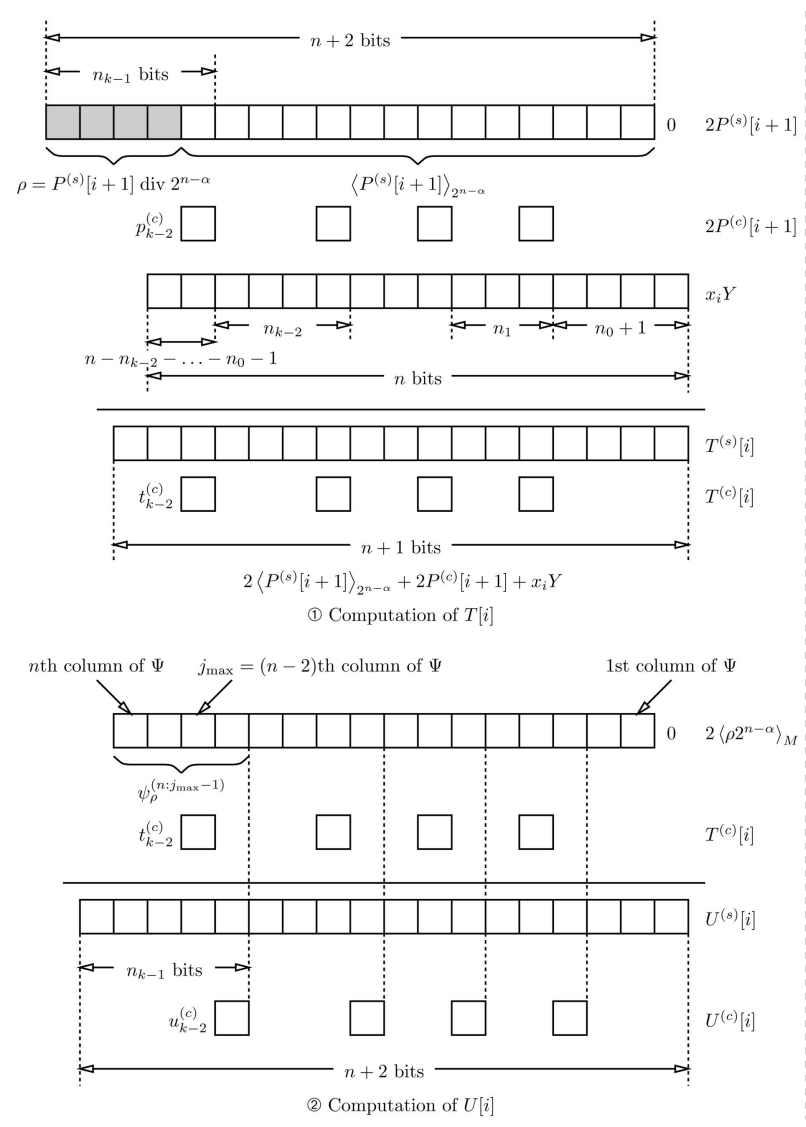

(a)

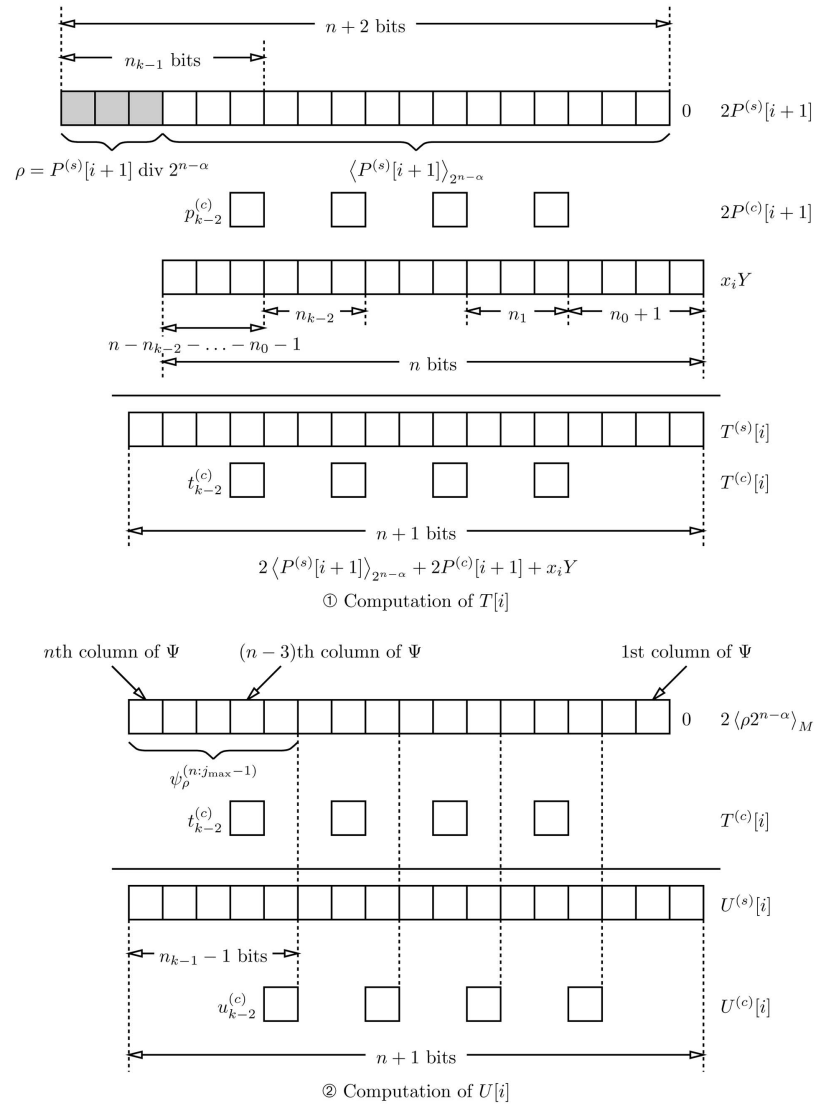

(b)

Fig. 7. Cost of the addition of a carry bit (3). (a) Computation of $U[i]$ when $\alpha=2$ and $j_{\max }=n-2$. (b) Computation of $U[i]$ when $\alpha=1$ and $j_{\max }=n-3$.

(Fig. 7a). The most significant sum word of $U[i]$ is computed as follows:

$$
u_{k-1}^{(s)}=2\left(\psi_{\rho}^{\left(n: j_{\max }\right)}+t_{k-2}^{(c)}\right)+\psi_{\rho}^{\left(j_{\max }-1: j_{\max }-1\right)} .
$$

When $\alpha=1$, we have $n_{k-1} \geq 6$ and $j_{\max }=n-3$ (Fig. 7b). It is sometimes possible to relax the constraint on $n_{k-1}$ : it suffices that the addition of $t_{k-2}^{(c)}$ to $\psi_{\rho}^{\left(n: j_{\max }\right)}$ does not generate an output carry (see the proof in Appendix A for details). This condition is satisfied if the length of the longest string of consecutive ones starting from the $j_{\max }$ column of $\Psi$ is smaller than or equal to $n-j_{\max }$. We have to distinguish three cases to build the graph:

- The first carry bit $t_{0}^{(c)}$ can be added to any column whose index belongs to $\left\{2, \ldots, w_{\max }+1\right\}$. We create an edge of weight zero between the first node and the nodes associated with such columns.

- The most significant carry bit $t_{k-2}^{(c)}$ belongs to the set $\left\{n-w_{\max }+1, \ldots, j_{\max }\right\}$. Let $h \in\left\{w_{\min }, \ldots, w_{\max }-1\right\}$. There is therefore an edge between nodes $j$ and $n$ if $j+h \geq n$.

- There is an edge between nodes $j$ and $j+h$, where $h \in\left\{w_{\min }, \ldots, w_{\max }\right\}$, if $j+h \leq j_{\max }$.

The next step consists of finding a shortest path in the graph. In order to minimize the critical path, we suggest to remove all edges whose carry propagation flag is set to one.
If there is no path between nodes 1 and $n$ in this pruned graph, we have to consider the full graph.

Algorithm 2. Selection of a high-radix carry-save number system.

Input: An $n$-bit modulus $M, w_{\max }$, and $w_{\min }$ such that $w_{\max } \geq w_{\min } \geq 2$. A parameter $\alpha \in\{1,2\}$.

Output: A directed acyclic graph.

1: Compute the matrix $\Psi$ according to $\alpha$;

2: if $\alpha=2$ then

3: $j_{\max } \leftarrow n-2$;

4: else

5: $\quad j_{\max } \leftarrow n-3$;

6: $j \leftarrow n-2$

7: $\quad$ while $\#\left(\psi_{\rho}^{(n: j)}\right) \leq n-j$ do

8: $\quad j_{\max } \leftarrow j$;

9: $\quad j \leftarrow j+1$;

10: end while

11: end if

12: for $j=2$ to $w_{\max }$ do

13: Create an edge of weight 0 between nodes 1 and $j$;

14: end for

15: for $j=2$ to $j_{\max }$ do

16: for $h=w_{\min }$ to $w_{\max }$ do

17: $\quad$ if $j+h \geq n$ and $h<w_{\max }$ then

18: $\quad \ell \leftarrow \max _{\rho} \#\left(\psi_{\rho}^{(n: j)}\right)$;

19: $\quad$ Create an edge between nodes $j$ and $n$; 


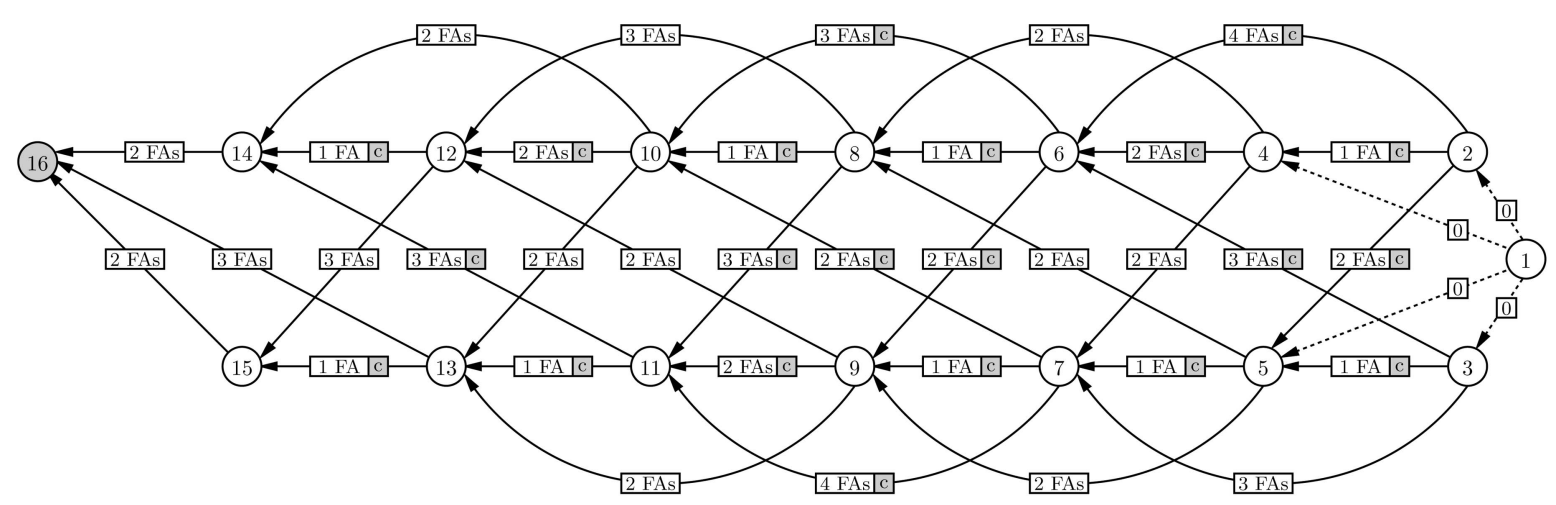

Fig. 8. Construction of a graph to select a high-radix carry-save representation for the 16-bit modulus $M=54,107$ (1).

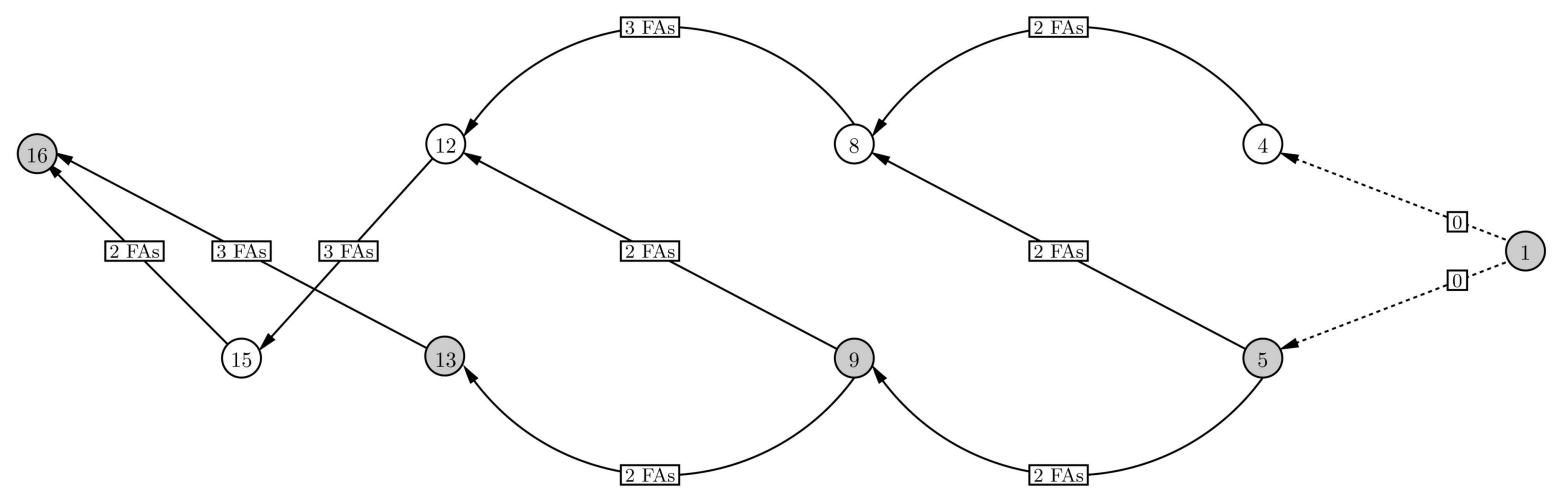

Fig. 9. Construction of a graph to select a high-radix carry-save representation for the 16-bit modulus $M=54,107$ (2). Shaded nodes belong to the shortest path.

20: $\quad$ Compute the weight of the edge (see Fig. 6);

21: $\quad h \leftarrow w_{\max }+1$ (exit the loop);

22: $\quad$ else if $j+h \leq j_{\max }$ then

23: $\quad \ell \leftarrow \max _{\rho} \#\left(\psi_{\rho}^{(j+h-2: j)}\right)$;

24: $\quad$ Create an edge between nodes $j$ and $j+h$;

25: Compute the weight of the edge (see Fig. 6);

26: end if

27: end for

28: end for

Example 6 (Example 4 continued). Let us apply Algorithm 2 to our 16-bit example. First, we note that adding a carry bit to the 15th column of the matrix does not generate an output carry, and we set $j_{\max }=15$. Then, we build the graph illustrated in Fig. 8 according to Algorithm 2. The $c$ flag on the edge between nodes $j$ and $j+h$ indicates that adding a carry bit to the $j$ th column of $\Psi$ generates an output carry bit $u_{j}^{(c)}$. After having removed all edges labeled with a $c$ flag and nodes without a predecessor or successor, we obtain a pruned graph (Fig. 9). Thus, $P[i]$ is a four-digit word with $n_{0}=n_{1}=n_{2}=4$ and $n_{3}=6$ (Fig. 10). Since $n=16$ and $\psi_{\max }=(1010110010100101)_{2}=44,197$, we have

$$
\begin{aligned}
& \frac{2^{n-1}-1+2^{n_{0}}+2^{n_{0}+n_{1}}+2^{n_{0}+n_{1}+n_{2}}+\psi_{\max }}{2} \\
& \quad=\frac{2^{15}+2^{4}+2^{8}+2^{12}+44,197}{2}=40,666<M,
\end{aligned}
$$

and $\alpha=1$ is a valid choice. Note that if the above equality is not satisfied, we have to build a new graph with $\alpha=2$. Recall that there is always a solution for $\alpha=2$ and $n_{k-1} \geq 5$.

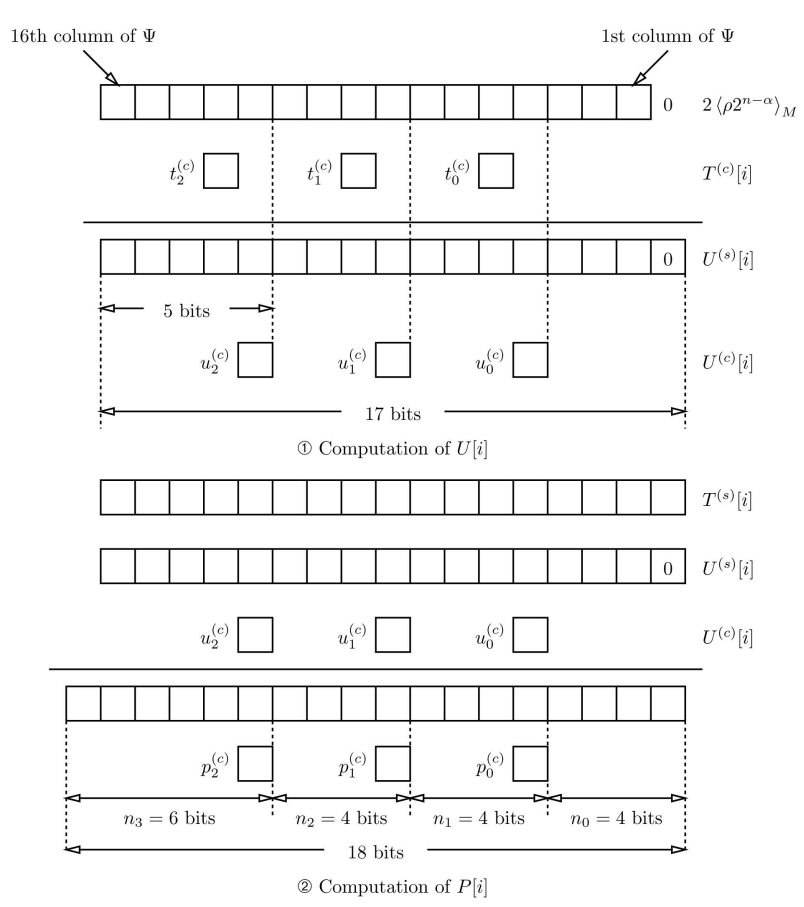

Fig. 10. Choice of a high-radix carry-save representation for the 16-bit modulus $M=54,107$ (3). 
Once the high-radix carry-save representation is known, the automatic generation of a VHDL description of the modulo $M$ multiplier is rather straightforward. The computation of $T[i]$ involves $k$ CRAs of respective widths $n_{0}+1, n_{1}, \ldots, n_{k}-2$, and $n-n_{k-2}-\cdots-n_{0}-1$ (Fig. 7). Each edge of the graph encodes the size of the CRA determining a digit of $U[i]$, and the carry propagation flag indicates whether a carry bit $u_{j}^{(c)}$ is necessary or not. Finally, $k$ CRAs of widths $n_{0}, \ldots, n_{k}-1$ allow one to add $T^{(s)}[i]$ to $U[i]$.

\section{Implementation Results}

In order to compare our algorithm against modular multipliers published in the open literature, we wrote a VHDL code generator whose inputs are a modulus $M$ and $w_{\max }$ (maximal number of positions between two consecutive carry bits; see Section 4). Our tool returns a structural VHDL description of a high-radix carry-save multiplier, as well as scripts to automatically place and route the design and collect area and timing information. This tool also generates a VHDL description of two architectures proposed by other researchers. The first one, described by Peeters et al. in [10], is summarized by Algorithm 3 . Intermediate results are carry-save numbers denoted by $(C[i], S[i])$. At each step, a CRA computes the sum $k$ of the three most significant bits of $C[i+1]$ and $S[i+1]$. This 4-bit word addresses a table storing $\left\langle k \cdot 2^{n-2}\right\rangle_{M}, 0 \leq k \leq 15$. Thanks to an additional iteration with $x_{-1}=0$, this algorithm returns a carry-save number $(C[-1], S[-1])$, which is smaller than $2 M$. Since our multiplier satisfies the same property, conversion in a nonredundant number system is performed with the same operator. ${ }^{2}$ We will therefore only consider iteration stages in our experiments in order to compare high-radix carry-save and carry-save number systems.

Algorithm 3. Peeters et al.'s modulo $M$ multiplication [10].

Input: An $n$-bit modulus $M$ such that $2^{n-1}<M<2^{n}$, an $r$-bit number $X \in \mathbb{N}$, and $Y \in\{0, \ldots, M-1\}$. We assume that $x_{-1}=0$.

Output: $P=\langle X Y\rangle_{M}$.

1. $C[r] \leftarrow 0 ; S[r] \leftarrow 0$;

2. for $i$ in $r-1$ downto -1 do

3. $k \leftarrow C[i+1] \operatorname{div} 2^{n-2}+S[i+1] \operatorname{div} 2^{n-2}$;

4. $T[i] \leftarrow x_{i} Y+2\left\langle k \cdot 2^{n-2}\right\rangle_{M}$;

5. $(C[i], S[i]) \leftarrow 2\left(\langle C[i+1]\rangle_{2^{n-2}}+\langle S[i+1]\rangle_{2^{n-2}}\right)+T[i] ;$

6. end for

7. $P \leftarrow(S[-1]+C[-1]) / 2$;

8. if $P>M$ then

9. $P \leftarrow P-M$;

10. end if

Amanor et al. introduced a carry-save architecture optimized for modular multiplication on FPGAs in [13]. The authors assume that both $M$ and $Y$ are known at design time. This hypothesis allows for the design of an iteration stage embedding a single CSA and a table addressed by the most significant bit of $x_{i}, C[i+1]$, and

2. Our approach further reduces the wiring since high-radix carry-save numbers involve less carry bits than carry-save numbers.
$S[i+1]$ (Algorithm 4). They show that the sum of the most significant bits of $C[i+1]$ and $S[i+1]$ is always a 2-bit number. Therefore, the table only contains eight values. Unfortunately, the authors did not address the final conversion issue. However, since $C[i+1] \operatorname{div} 2^{n-1}+$ $S[i+1] \operatorname{div} 2^{n-1} \leq 3$, we deduce that

$$
\begin{aligned}
C[i]+S[i]= & \left(C[i+1] \operatorname{div} 2^{n-1}+S[i+1] \operatorname{div} 2^{n-1}\right) \cdot 2^{n-1} \\
& +\langle C[i+1]\rangle_{2^{n-1}}+\langle S[i+1]\rangle_{2^{n-1}} \\
\leq & 3 \cdot 2^{n-1}+2 \cdot\left(2^{n-1}-1\right)=2^{n+1}+2^{n-1}-2 .
\end{aligned}
$$

Since $M$ belongs to $\left\{2^{n-1}+1, \ldots, 2^{n}-1\right\}, C[i]+S[i]$ may be greater than $2 M$, and Algorithm 4 requires more hardware resources than our algorithm or Peeters et al.'s scheme to perform a conversion.

Algorithm 4. Amanor et al.'s modulo $M$ multiplication [13]. Input: An $n$-bit modulus $M$ such that $2^{n-1}<M<2^{n}$, an $r$-bit number $X \in \mathbb{N}$, and $Y \in \mathbb{N}$.

Output: $P=\langle X Y\rangle_{M}$.

1. $C[r] \leftarrow 0 ; S[r] \leftarrow 0$;

2. for $i$ in $r-1$ downto 0 do

3. $k \leftarrow 2\left(C[i+1] \operatorname{div} 2^{n-1}+S[i+1] \operatorname{div} 2^{n-1}\right)$;

4. $T[i] \leftarrow\left\langle k \cdot 2^{n-1}+x_{i} Y\right\rangle_{M}$;

5. $(C[i], S[i]) \leftarrow 2\left(\langle C[i+1]\rangle 2^{n-1}+\langle S[i+1]\rangle 2^{n-1}\right)+T[i] ;$

6. end for

7. $P \leftarrow\langle C[0]+S[0]\rangle_{M}$;

Fig. 11 describes place-and-route results on a Xilinx Spartan-3 FPGA. In these experiments, the moduli are 256-bit randomly generated primes. Compared against Algorithm 3, we observe the following:

- Our high-radix carry-save architecture allows us to significantly reduce the number of slices while only slightly augmenting the critical path. At the price of a longer critical path, we are able to further diminish the area by increasing the parameter $w_{\max }$. Note that conversion from (high-radix) carry-save to unsigned binary integer is usually based on pipelined CRAs (see, for instance, [3]). Depending on the trade-off between area and delay, this operator can be slower than an iteration stage based on (high-radix) carrysave arithmetic.

- The area of our operator is less sensitive to the choice of $M$. This is mainly related to the architecture of Xilinx FPGAs: in most cases, $\alpha=1$, and each operator embeds a table addressed by 3 bits. Since our target FPGA embeds four-input LUTs, this table is embedded within the slices of the adder computing $P[i]$ [11]. Since 4 bits address the table of Algorithm 3, additional LUTs are requested. Their amount depends on the modulus $M$ : if $\psi_{M}$ contains null or identical columns, synthesis tools are able to simplify the design.

For the moduli considered in these experiments, highradix carry-save multipliers have roughly the same area as the operator proposed by Amanor et al. in [13]. Recall that a CSA requires twice the number of slices of a CRA 
Proposed algorithm; $\boldsymbol{\nabla}$ Algorithm 3; $\times$ Algorithm 4.
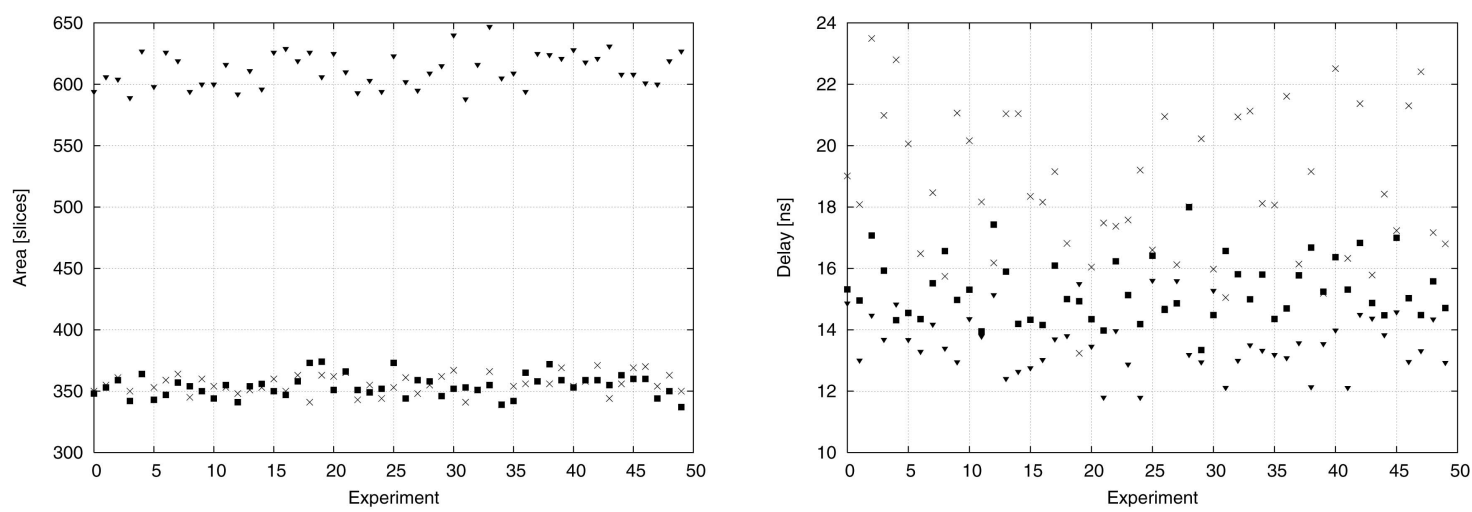

(a)
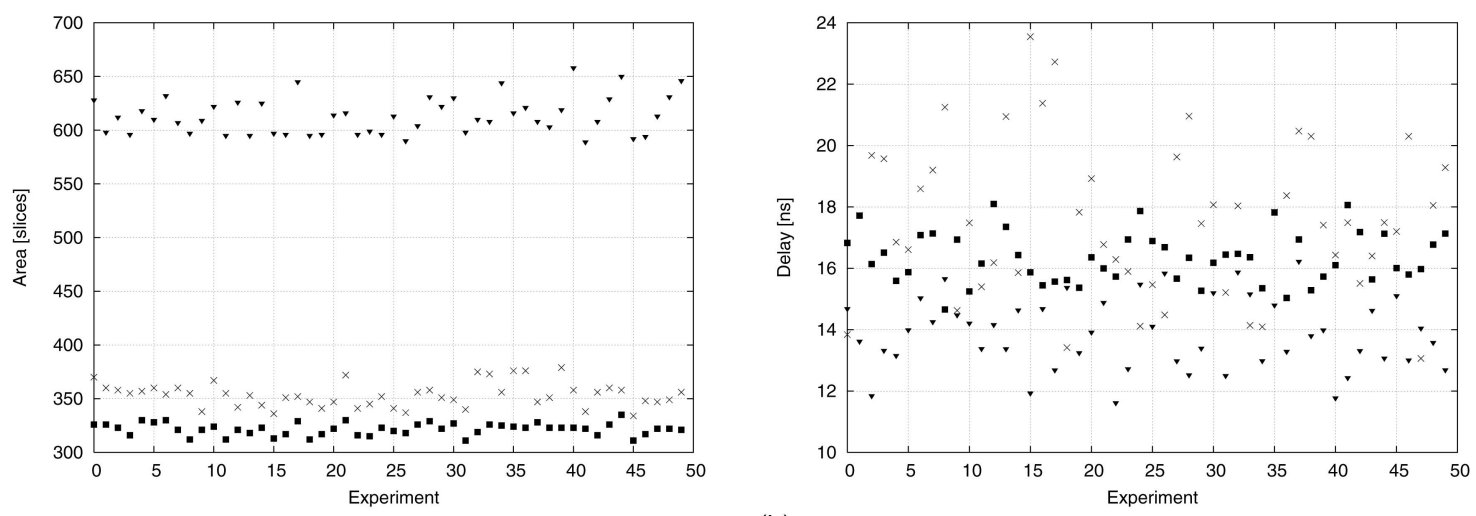

(b)
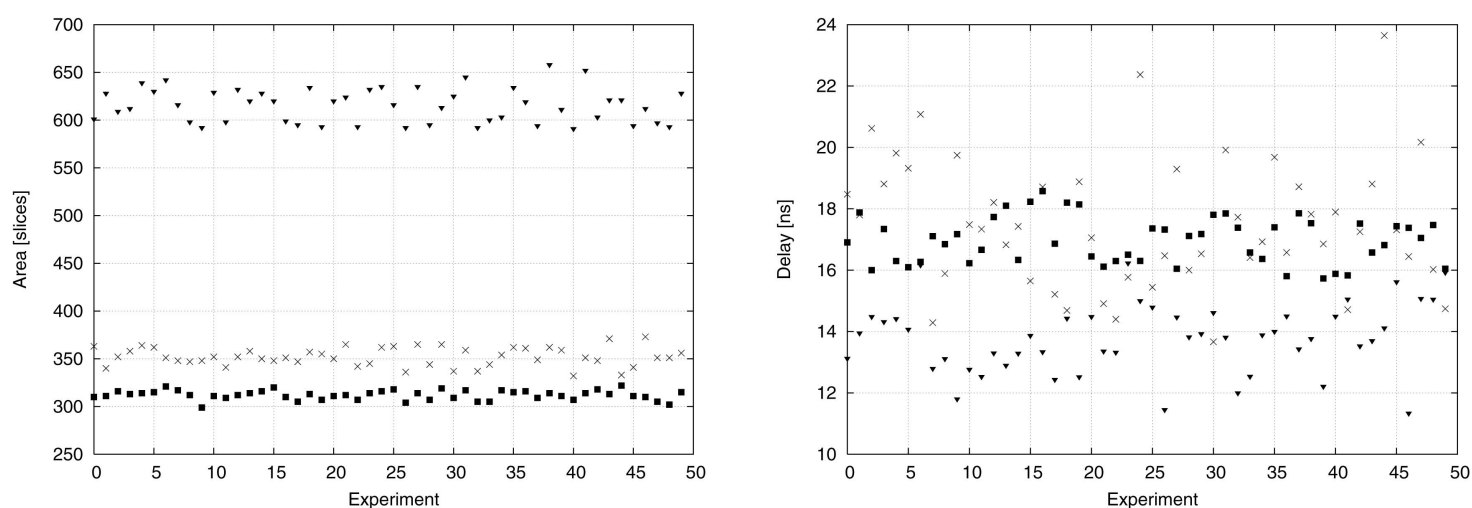

(c)


(d)

Fig. 11. Area and delay of modular multipliers on a Spartan-3 FPGA. Fifty prime moduli were randomly generated for each experiment. (a) Area and delay comparisons for $n=256$ and $w_{\max }=8$. (b) Area and delay comparisons for $n=256$ and $w_{\max }=16$. (c) Area and delay comparisons for $n=256$ and $w_{\max }=24$. (d) Area and delay comparisons for $n=256$ and $w_{\max }=32$. 
TABLE 3

Area and Delay Ratios between Our Proposal and Algorithms 3 and 4 on a Spartan-3 FPGA

\begin{tabular}{|c|c|c|c|c|c|}
\hline \multirow[b]{2}{*}{$\mathbf{N}$} & \multirow[b]{2}{*}{$\mathbf{w}_{\text {max }}$} & \multicolumn{2}{|c|}{ Peeters et al. $[10]$} & \multicolumn{2}{|c|}{ Amanor et al. [13] } \\
\hline & & $\begin{array}{l}\text { Area of Algorithm I } \\
\text { Area of Algorithm } 3\end{array}$ & $\begin{array}{l}\text { Delay of Algorithm I } \\
\text { Delay of Algorithm } 3\end{array}$ & $\begin{array}{l}\text { Area of Algorithm I } \\
\text { Area of Algorithm } 4 \\
\end{array}$ & $\begin{array}{l}\text { Delay of Algorithm } 1 \\
\text { Delay of Algorithm } 4\end{array}$ \\
\hline \multirow{2}{*}{64} & 8 & {$[0.45,0.68]$} & {$[0.89,1.45]$} & {$[0.77,1.12]$} & {$[1.10,1.62]$} \\
\hline & 16 & {$[0.39,0.58]$} & {$[0.97,1.49]$} & {$[0.73,0.97]$} & {$[1.12,1.84]$} \\
\hline \multirow{4}{*}{128} & 8 & {$[0.48,0.68]$} & {$[0.99,1.32]$} & {$[0.89,1.28]$} & {$[0.99,1.38]$} \\
\hline & 16 & {$[0.44,0.55]$} & {$[0.99,1.32]$} & {$[0.79,0.96]$} & {$[0.99,1.44]$} \\
\hline & 32 & {$[0.43,0.53]$} & {$[1.00,1.44]$} & {$[0.77,0.91]$} & {$[1.01,1.61]$} \\
\hline & 48 & {$[0.40,0.50]$} & {$[1.04,1.69]$} & {$[0.74,0.89]$} & {$[1.11,1.79]$} \\
\hline \multirow{4}{*}{160} & 8 & {$[0.51,0.64]$} & {$[0.98,1.15]$} & {$[0.90,1.09]$} & {$[0.99,1.23]$} \\
\hline & 16 & {$[0.48,0.56]$} & {$[0.99,1.19]$} & {$[0.84,0.96]$} & {$[0.99,1.32]$} \\
\hline & 32 & {$[0.44,0.53]$} & {$[1.04,1.36]$} & {$[0.78,0.94]$} & {$[1.04,1.45]$} \\
\hline & 48 & {$[0.36,0.52]$} & {$[1.11,1.56]$} & {$[0.79,0.89]$} & {$[1.18,1.63]$} \\
\hline \multirow{4}{*}{192} & 8 & {$[0.53,0.63]$} & {$[0.57,1.38]$} & {$[0.92,1.12]$} & {$[0.67,1.49]$} \\
\hline & 16 & {$[0.48,0.55]$} & {$[0.62,1.47]$} & {$[0.82,0.97]$} & {$[0.82,1.59]$} \\
\hline & 24 & {$[0.46,0.53]$} & {$[0.55,1.49]$} & {$[0.83,0.98]$} & {$[0.85,1.69]$} \\
\hline & 32 & {$[0.46,0.52]$} & {$[0.65,1.46]$} & {$[0.79,0.93]$} & {$[0.94,1.66]$} \\
\hline \multirow{4}{*}{256} & 8 & {$[0.54,0.63]$} & {$[0.89,1.48]$} & {$[0.93,1.11]$} & {$[0.59,1.27]$} \\
\hline & 16 & {$[0.49,0.55]$} & {$[0.94,1.45]$} & {$[0.85,0.98]$} & {$[0.67,1.34]$} \\
\hline & 24 & {$[0.48,0.53]$} & {$[0.99,1.59]$} & {$[0.83,0.97]$} & {$[0.71,1.38]$} \\
\hline & 32 & {$[0.47,0.53]$} & {$[1.01,1.68]$} & {$[0.79,0.94]$} & {$[0.66,1.35]$} \\
\hline
\end{tabular}

One hundred prime moduli were randomly generated for each experiment. We report the intervals in which lie the area and delay ratios.

TABLE 4

Area and Delay Ratios between Our Proposal and Algorithms 3 and 4 on a Cyclone II FPGA

\begin{tabular}{|c|c||c|c||c|c|}
\hline \multirow{2}{*}{$\mathbf{N}$} & \multirow{2}{*}{$\mathbf{w}_{\max }$} & \multicolumn{2}{c||}{ Peeters et al. [10] } & \multicolumn{2}{c|}{ Amanor et al. [13] } \\
\cline { 3 - 6 } & & $\frac{\text { Area of Algorithm 1 }}{\text { Area of Algorithm } \mathbf{3}}$ & $\frac{\text { Delay of Algorithm 1 }}{\text { Delay of Algorith } \mathbf{3}}$ & $\frac{\text { Area of Algorithm 1 } 1}{\text { Area of Algorithm } \mathbf{4}}$ & $\frac{\text { Delay of Algorithm I }}{\text { Delay of Algorithm } \mathbf{4}}$ \\
\hline \hline \multirow{2}{*}{$\mathbf{6 4}$} & $\mathbf{8}$ & {$[0.63,0.77]$} & {$[1.45,1.87]$} & {$[1.14,1.36]$} & {$[1.92,2.54]$} \\
\cline { 2 - 6 } & $\mathbf{1 6}$ & {$[0.60,0.66]$} & {$[1.58,2.02]$} & {$[1.06,1.19]$} & {$[2.18,2.75]$} \\
\hline \hline \multirow{3}{*}{$\mathbf{1 2 8}$} & $\mathbf{8}$ & {$[0.67,0.74]$} & {$[1.42,1.87]$} & {$[1.14,1.30]$} & {$[1.77,2.49]$} \\
\cline { 2 - 6 } & $\mathbf{1 6}$ & {$[0.62,0.65]$} & {$[1.63,2.04]$} & {$[1.07,1.15]$} & {$[2.09,2.85]$} \\
\cline { 2 - 6 } & $\mathbf{3 2}$ & {$[0.58,0.63]$} & {$[1.85,2.73]$} & {$[1.01,1.09]$} & {$[2.18,3.79]$} \\
\hline
\end{tabular}

One hundred prime moduli were randomly generated for each experiment. We report the intervals in which lie the area and delay ratios.

on our target FPGA family. Since the moduli involved in these experiments require only 3 bits to perform a modulo $M$ reduction, our architecture is mainly based on two CRAs. High-radix carry-save representations enable here the design of a more versatile modular multiplier (both $X$ and $Y$ are inputs) with the same number of slices.

Table 3 summarizes further results obtained with a Spartan-3 FPGA. We generated 100 prime moduli for each experiment and reported the intervals in which lie the area and delay ratios between our proposal and Algorithms 3 and 4. These experiments indicate that our approach always allows one to select a prime number that reduces the circuit area without increasing the critical path.

Table 4 digests experiment results involving an Altera Cyclone II FPGA. Fig. 12 describes a Logic Element (LE), which is the smallest unit of configurable logic in the Cyclone II architecture. Each LE includes a four-input LUT, a storage element, and dedicated carry logic and operates in normal mode or arithmetic mode. CRAs are based on LEs in arithmetic mode, in which the LUT implements two three-input function generators. It is therefore impossible to store $\psi_{M}$ within LUTs of a CRA. This explains why our algorithm leads to slightly smaller



Fig. 12. Simplified diagram of an LE in arithmetic mode (Cyclone II family). 
area savings for this FPGA family. On Cyclone-II devices, CSA operators are significantly faster; however, conversion to a nonredundant number system involves pipelined CRAs. If this operator is based on 32-bit blocks, our high-radix carry-save iteration stage has a slower critical path. In this case, our approach leads to smaller modular multipliers than CSA schemes, without impacting on computation time.

\section{Conclusion}

We proposed an algorithm to automatically generate VHDL descriptions of modular multipliers for FPGAs. The main originality of our approach is the selection of an optimal high-radix carry-save encoding of intermediate results according to a given modulus $M$. High-radix carry-save number systems take advantage of dedicated carry logic available in almost all FPGA families and reduce the amount of interconnects. Therefore, our approach allows us to significantly reduce the area of modular multipliers.

\section{APPENDIX A}

This appendix aims at proving the correctness of Algorithm 1. We proceed in three steps: after establishing a property of the modulo $M$ correction considered in this paper, we show that $P^{(s)}[i]$ is an $(n+2)$-bit number. We conclude by computing a bound on $P[-1]$, which indicates that $P[-1] / 2<2 M$. This proof also provides the reader with all the technical details requested to implement the algorithm or an automatic code generator.

\section{A.1 A Property of Modulo $M$ Correction}

The first step consists of establishing a property that will allow us to compute bounds on $P[i]$. Let $\gamma=2^{n}-2^{n-4}=$ $2^{n-1}+2^{n-2}+2^{n-3}+2^{n-4}$ and $M \in\left\{2^{n-1}, \ldots, 2^{n}-1\right\}$. Then

$$
\left\langle k \cdot 2^{n-2}\right\rangle_{M}<\gamma, \forall k \in 0, \ldots, 2^{4}-1 .
$$

The proof is straightforward if the modulus $M$ is smaller than or equal to $\gamma$. Let us assume now that $M=\gamma+\beta$, where $\beta$ satisfies the following inequality:

$$
1 \leq \beta \leq 2^{n-4}-1 .
$$

For $k \in\{0,1,2,3\}$, we easily check that $\left\langle k \cdot 2^{n-2}\right\rangle_{M}=$ $k \cdot 2^{n-2}<\gamma$. Since $\left\langle 2^{n}\right\rangle_{M}=2^{n}-M$, we obtain

$$
\left\langle 4 \cdot 2^{n-2}\right\rangle_{M}=2^{n-4}-\beta<\gamma .
$$

Consequently, we have

$$
\begin{aligned}
& \left\langle 5 \cdot 2^{n-2}\right\rangle_{M}=2^{n-2}+2^{n-4}-\beta<\gamma, \\
& \left\langle 6 \cdot 2^{n-2}\right\rangle_{M}=2^{n-1}+2^{n-4}-\beta<\gamma, \\
& \left\langle 7 \cdot 2^{n-2}\right\rangle_{M}=2^{n-1}+2^{n-2}+2^{n-4}-\beta<\gamma .
\end{aligned}
$$

For $k=8$, the following modulo $M$ operation has to be carried out:

$$
\left\langle 8 \cdot 2^{n-2}\right\rangle_{M}=\left\langle 2^{n}+2^{n-4}-\beta\right\rangle_{M} .
$$

Since $M<2^{n}+1 \leq 2^{n}+2^{n-4}-\beta \leq 2^{n}+2^{n-4}-1<2 M$, we deduce that

$$
\left\langle 8 \cdot 2^{n-2}\right\rangle_{M}=2^{n}+2^{n-4}-\beta-M=2^{n-3}-2 \beta .
$$

Thus, we have

$$
\begin{aligned}
\left\langle 9 \cdot 2^{n-2}\right\rangle_{M} & =2^{n-2}+2^{n-3}-2 \beta<\gamma, \\
\left\langle 10 \cdot 2^{n-2}\right\rangle_{M} & =2^{n-1}+2^{n-3}-2 \beta<\gamma, \\
\left\langle 11 \cdot 2^{n-2}\right\rangle_{M} & =2^{n-1}+2^{n-2}+2^{n-3}-2 \beta<\gamma .
\end{aligned}
$$

A modulo $M$ reduction is again required for $k=12$. Since

$$
M<2^{n}+2 \leq 2^{n}+2^{n-3}-2 \beta \leq 2^{n}+2^{n-3}-2<2 M,
$$

we obtain

$$
\begin{aligned}
\left\langle 12 \cdot 2^{n-2}\right\rangle_{M} & =\left\langle 2^{n}+2^{n-3}-2 \beta\right\rangle_{M} \\
& =2^{n}+2^{n-3}-2 \beta-M \\
& =2^{n-3}+2^{n-4}-3 \beta .
\end{aligned}
$$

Since $\beta \geq 1$, we conclude the proof by noting that

$$
\begin{aligned}
& \left\langle 13 \cdot 2^{n-2}\right\rangle_{M}=2^{n-2}+2^{n-3}+2^{n-4}-3 \beta<\gamma, \\
& \left\langle 14 \cdot 2^{n-2}\right\rangle_{M}=2^{n-1}+2^{n-3}+2^{n-4}-3 \beta<\gamma, \\
& \left\langle 15 \cdot 2^{n-2}\right\rangle_{M}=2^{n-1}+2^{n-2}+2^{n-3}+2^{n-4}-3 \beta<\gamma .
\end{aligned}
$$

\section{A.2 Width of $P^{(s)}[i]$}

Let us prove by induction that $P[i]$ is an $(n+2)$-bit number. Since $P[r]=0$, we check that $k=0$, and $T[r-1]=$ $P[r-1]=x_{r-1} Y$, which is an $n$-bit number. This property holds for $i=r-1$. Assume now that $P^{(s)}[i+1]$ is an $(n+2)$-bit number. We have to consider two cases according to the parameter $\alpha$ :

- Our hypotheses guarantee that $n_{k-1} \geq 5$ for $\alpha=2$. Therefore, $2\left\langle P^{(s)}[i+1]\right\rangle_{2^{n-2}}$ contains $k$ sum words of respective widths $n_{0}^{\prime}=n_{0}+1, \ldots, n_{k-2}^{\prime}=n_{k-2}$, and $n_{k-1}^{\prime}=n_{k-1}-4$ (Fig. 13a). Let us split the partial product $x_{i} Y$ into $k$ blocks in order to add it word by word to $2\left\langle P^{(s)}[i+1]\right\rangle_{2^{n-2}}$. We know that

$$
\sum_{i=0}^{k-1} n_{i}^{\prime}=n-1 \text {. }
$$

Since $x_{i} Y$ is an $n$-bit integer, we deduce from the above equation that its most significant sum word contains $n_{k-1}^{\prime \prime}=n_{k-1}^{\prime}+1=n_{k-1}-3$ bits. Therefore, the sum of the most significant bits of $2\left\langle P^{(s)}[i+1]\right\rangle_{2^{n-2}}, x_{i} Y$, and a carry bit is bounded by

$$
\begin{aligned}
\left(2^{n_{k-1}^{\prime}+1}-1\right)+\left(2^{n_{k-1}^{\prime}}-1\right)+1 & =2^{n_{k-1}-3}+2^{n_{k-1}-4}-1 \\
& =3 \cdot 2^{n_{k-1}-4}-1,
\end{aligned}
$$

which is an $\left(n_{k-1}-2\right)$ bit number. Therefore, since $\sum_{i=0}^{k-1} n_{i}=n+2, T^{(s)}[i]$ is an $(n+1)$-bit number. Indeed, we have

$$
\left(n_{k-1}-2\right)+\sum_{i=1}^{k-2} n_{i}+\left(n_{0}+1\right)=\sum_{i=0}^{k-1} n_{i}-1
$$$$
=n+1 \text {. }
$$ 


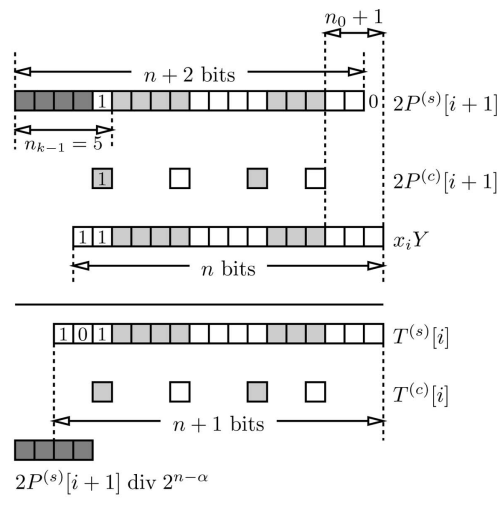

(a)

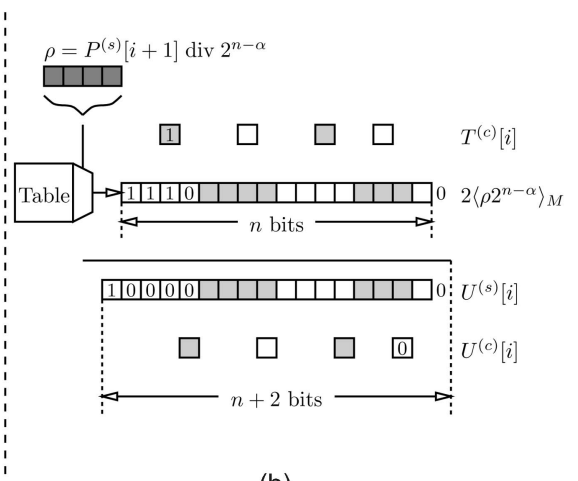

(b)

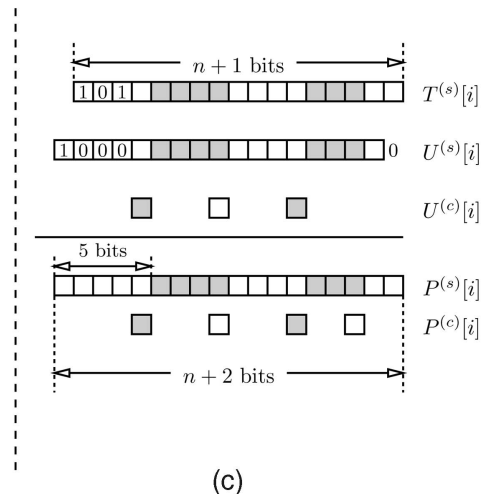

(c)

Fig. 13. Proof of Algorithm 1 for $\alpha=2$. (a) Computation of $T[i]$. (b) Modulo $M$ reduction and conversion. (c) Computation of $P[i]$.

Four most significant bits of $P^{(s)}[i+1]$ address the table responsible for the modulo $M$ correction (Fig. 13b). Recall that we have to combine the output of this table and carry bits of $T[i]$ in order to generate a high-radix carry-save number $U[i]$, whose format is the one of $P[i]$. Since $2\left\langle k \cdot 2^{n-\alpha}\right\rangle_{M}$ is an $(n+1)$-bit number, we split it into $k$ words of respective lengths $n_{0}, n_{1}, \ldots, n_{k-2}$, and $\left(n_{k-1}-1\right)$. Consider now the addition of the most significant words of $2\left\langle k \cdot 2^{n-\alpha}\right\rangle_{M}$ and $T^{(s)}[i]$ and the most significant carry bit of $T^{(c)}[i]$. According to our hypotheses, $n_{k-1} \geq 5$, and this most significant word contains at least 4 bits. Consider the worst case (Fig. 13b), where $n_{k-1}=5$ and the weight of the most significant bit of $T^{(c)}[i]$ is equal to $2^{n-2}$. We deduce from (9) that $U^{(s)}[i]$ is an $(n+2)$-bit number and that its most significant sum word is smaller than or equal to $2^{4}$. The addition of the most significant words of $T^{(s)}[i]$ and $U^{(s)}[i]$ and a carry bit never generates an output carry, and $P^{(s)}[i]$ is therefore an $(n+2)$-bit number (Fig. 13c).

- Assume now that $\alpha=1$. The same approach allows us to show that $T^{(s)}[i]$ is an $(n+1)$-bit word (Fig. 14a). According to our hypotheses, the most significant word of $P^{(s)}[i-1]$ contains at least 6 bits. Therefore, the weight of the most significant carry bit of $T^{(c)}[i]$ is at most $2^{n-3}$. Since (9) guarantees that $2\left\langle k \cdot 2^{n-\alpha}\right\rangle_{M}<2^{n}+2^{n-1}+2^{n-2}+2^{n-3}$, we deduce that $U^{(s)}[i]$ is an $(n+1)$-bit number (Fig. 14b). Note that for some moduli, we can relax the constraint on $n_{k-1}$ : the remainder of the proof will only assume that $U^{(s)}[i]$ is an $(n+1)$-bit number. An automatic code generator can check this condition very easily for a given value of $M$. Since the most significant words of $T^{(s)}[i]$ and $U^{(s)}[i]$ have the same size, their addition may generate an output carry, and $P^{(s)}[i]$ is therefore an $(n+2)$-bit number (Fig. 14c).

\section{A.3 Final Modulo $M$ Correction}

The last step consists of proving that $P[-1] / 2$ is smaller than $2 M$. We have again to consider two cases according to $\alpha$ :

- Assume that $\alpha=2$ and consider the last iteration (i.e., $i=-1$ ). Since the partial product $x_{-1} Y$ is equal to zero, we have

$$
\begin{aligned}
T[-1] & =2 \cdot\left(\left\langle P^{(s)}[0]\right\rangle_{2^{n-2}}+P^{(c)}[0]\right) \\
& \leq 2 \cdot\left(2^{n-2}-1+2^{n-2}-1\right)=2^{n}-4 .
\end{aligned}
$$

Thus, $P[-1] \leq 2^{n}+2 M-6$, and $P[-1] / 2 \leq 2^{n-1}+$ $M-3$. Since the modulus $M$ is supposed to be greater than $2^{n-1}$, we know that $P[-1] / 2$ is smaller than $2 M$.

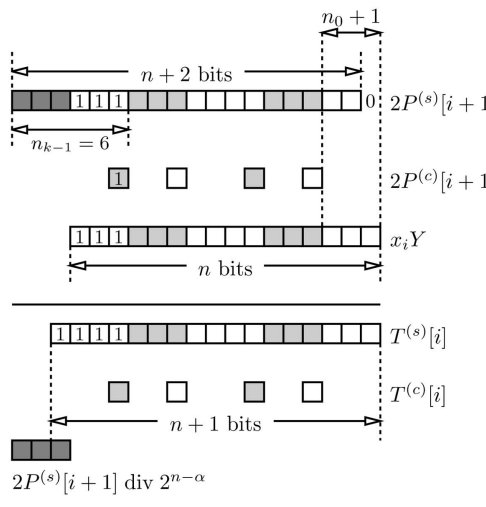

(a)

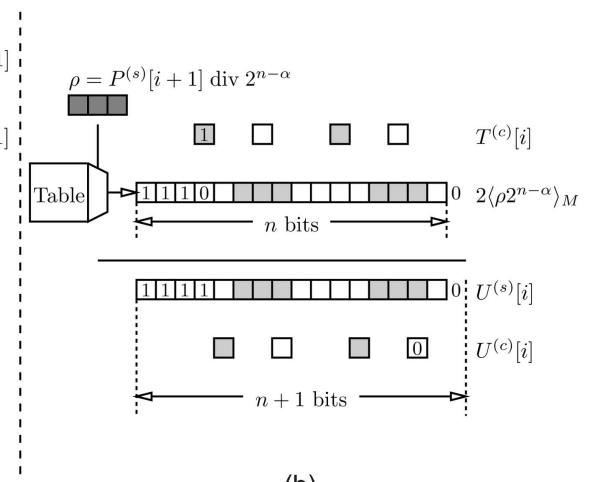

(b)

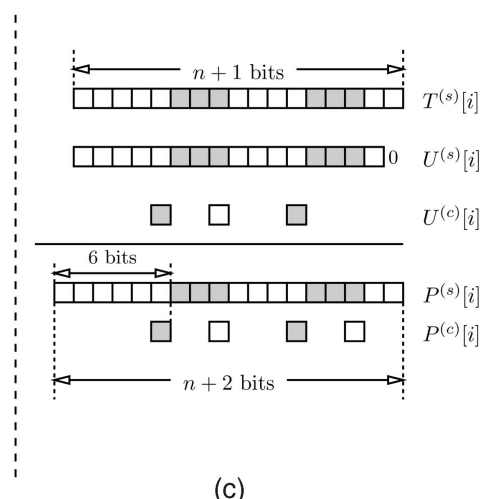

(c)

Fig. 14. Proof of Algorithm 1 for $\alpha=1$. (a) Computation of $T[i]$. (b) Modulo $M$ reduction and conversion. (c) Computation of $P[i]$. 
- When $\alpha=1,\left\langle P^{(s)}[i+1]\right\rangle_{2^{n-\alpha}}$ is smaller than or equal to $2^{n-1}-1$. Recall that the weight of the most significant carry bit of $P^{(c)}[i+1]$ is equal to $n_{0}+n_{1}+\cdots+n_{k-2}$ (Section 2). Thus

$$
T[-1] \leq 2^{n}-2+2^{n_{0}+1}+\cdots+2^{n_{0}+\cdots+n_{k-2}+1},
$$

and

$$
P[-1] \leq 2^{n}-2+2^{n_{0}+1}+\cdots+2^{n_{0}+\cdots+n_{k-2}+1}+2 \psi_{\max }
$$

Therefore, $P[-1] / 2$ is smaller than $2 M$ if

$$
\frac{2^{n-1}-1+2^{n_{0}}+\cdots+2^{n_{0}+\cdots+n_{k-2}}+\psi_{\max }}{2}<M .
$$

\section{ACKNOWLEDGMENTS}

The authors are grateful to the anonymous referees for their valuable comments. The work described in this paper has been supported in part by the New Energy and Industrial Technology Development Organization (NEDO), Japan, and by the Swiss National Science Foundation through the Advanced Researchers program while Jean-Luc Beuchat was at École Normale Supérieure de Lyon (Grant PA002101386). The authors would like to thank F. de Dinechin and J. Detrey for administrating the CAD tool servers on which experiments described in this paper were performed.

\section{REFERENCES}

[1] P. Montgomery, "Modular Multiplication without Trial Division," Math. of Computation, vol. 44, no. 170, pp. 519-521, 1985.

[2] G.R. Blakley, "A Computer Algorithm for Calculating the Product ab Modulo m," IEEE Trans. Computers, vol. 32, no. 5, pp. 497-500, May 1983.

[3] M.D. Ercegovac and T. Lang, Digital Arithmetic. Morgan Kaufmann, 2004.

[4] C.K. Koç and C.Y. Hung, "Carry-Save Adders for Computing the Product AB Modulo N," Electronics Letters, vol. 26, no. 13, pp. 899-900, June 1990.

[5] C.K. Koç and C.Y. Hung, "A Fast Algorithm for Modular Reduction," IEE Proc.: Computers and Digital Techniques, vol. 145, no. 4, pp. 265-271, July 1998.

[6] N. Takagi and S. Yajima, "Modular Multiplication Hardware Algorithms with a Redundant Representation and Their Application to RSA Cryptosystem," IEEE Trans. Computers, vol. 41, no. 7, pp. 887-891, July 1992.

[7] N. Takagi, "A Radix-4 Modular Multiplication Hardware Algorithm for Modular Exponentiation," IEEE Trans. Computers, vol. 41, no. 8, pp. 949-956, Aug. 1992.

[8] Y.-J. Jeong and W.P. Burleson, "VLSI Array Algorithms and Architectures for RSA Modular Multiplication," IEEE Trans. VLSI Systems, vol. 5, no. 2, pp. 211-217, June 1997.

[9] S. Kim and G.E. Sobelman, "Digit-Serial Modular Multiplication Using Skew-Tolerant Domino CMOS," Proc. IEEE Int'l Conf. Acoustics, Speech, and Signal Processing (ICASSP '01), vol. 2, pp. 1173-1176, 2001.

[10] E. Peeters, M. Neve, and M. Ciet, "XTR Implementation on Reconfigurable Hardware," Proc. Workshop Cryptographic Hardware and Embedded Systems (CHES '04), M. Joye and J.-J. Quisquater, eds., pp. 386-399, 2004.

[11] J.-L. Beuchat and J.-M. Muller, "Modulo $m$ MultiplicationAddition: Algorithms and FPGA Implementation," Electronics Letters, vol. 40, no. 11, pp. 654-655, May 2004.

[12] R. Beguenane, J.-L. Beuchat, J.-M. Muller, and S. Simard, "Modular Multiplication of Large Integers on FPGA," Proc. 39th Asilomar Conf. Signals, Systems and Computers, 2005.

[13] D.N. Amanor, C. Paar, J. Pelzl, V. Bunimov, and M. Schimmler, "Efficient Hardware Architectures for Modular Multiplication on FPGAs," Proc. 15th Int'l Conf. Field Programmable Logic and Applications (FPL '05), pp. 539-542, 2005.

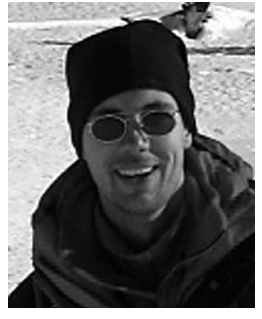

Jean-Luc Beuchat received the MSc and $\mathrm{PhD}$ degrees in computer science from the Swiss Federal Institute of Technology, Lausanne, in 1997 and 2001, respectively. He is an associate professor in the Graduate School of Systems and Information Engineering, Laboratory of Cryptography and Information Security, University of Tsukuba, Tsukuba, Japan. His current research interests include computer arithmetic and cryptography.

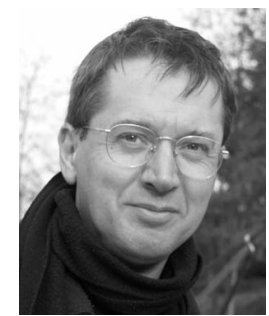

Jean-Michel Muller received the PhD degree from the Institut National Polytechnique de Grenoble in 1985. He is the directeur de recherches (senior researcher) at CNRS, France, and he is the former head of the LIP Laboratory (LIP is a joint laboratory of CNRS, the École Normale Supérieure de Lyon, INRIA, and the Université Claude Bernard Lyon 1). His research interests are in computer arithmetic. $\mathrm{He}$ is the author of several books, including Elementary Functions, Algorithms and Implementation (second edition, Birkhäuser, 2006). He served as an associate editor of the IEEE Transactions on Computers from 1996 to 2000. He was the co-program chair of the 13th IEEE Symposium on Computer Arithmetic in 1997 and the general chair of the 14th IEEE Symposium on Computer Arithmetic in 1999. He is a senior member of the IEEE and a member of the IEEE Computer Society.

$\triangleright$ For more information on this or any other computing topic, please visit our Digital Library at www.computer.org/publications/dlib. 